\title{
A RIEMANN-HILBERT PROBLEM FOR THE MOISIL-TEODORESCU SYSTEM
}

\author{
ALEXANDER POLKOVNIKOV AND NIKOLAI TARKHANOV
}

\begin{abstract}
In a bounded domain with smooth boundary in $\mathbb{R}^{3}$ we consider the stationary Maxwell equations for a function $u$ with values in $\mathbb{R}^{3}$ subject to a nonhomogeneous condition $(u, v)_{x}=u_{0}$ on the boundary, where $v$ is a given vector field and $u_{0}$ a function on the boundary. We specify this problem within the framework of the Riemann-Hilbert boundary value problems for the Moisil-Teodorescu system. This latter is proved to satisfy the ShapiroLopaniskij condition if an only if the vector $v$ is at no point tangent to the boundary. The Riemann-Hilbert problem for the Moisil-Teodorescu system fails to possess an adjoint boundary value problem with respect to the Green formula, which satisfies the Shapiro-Lopatinskij condition. We develop the construction of Green formula to get a proper concept of adjoint boundary value problem.
\end{abstract}

\section{CONTENTS}

Introduction 1

Part 1. The Riemann-Hilbert boundary value problem 3

1. Setting of the problem 3

2. The main theorem on elliptic boundary value problems 3

3. Applications to the Riemann-Hilbert problem 5

\begin{tabular}{|l|l|}
\hline Part 2. The Moisil-Teodoresco system & 11
\end{tabular}

4. The Green formula 11

5. A calculation for the Moisil-Teodoresco system 14

\begin{tabular}{lll}
\hline 6. & The Fredholm property & 16
\end{tabular}

\begin{tabular}{lll}
\hline Part 3. & Boundary Fourier method & 18
\end{tabular}

$\begin{array}{ll}\text { 7. Hardy spaces } & 18\end{array}$

8. The Cauchy problem 20

$\begin{array}{lll}9 . & \text { Operator-theoretic foundations } & 21\end{array}$

Part 4. Application to the Riemann-Hilbert problem 24

Date: April 12, 2017.

2010 Mathematics Subject Classification. Primary 35F45; Secondary 35J56, 47N20.

Key words and phrases. Dirac operator, Riemann-Hilbert problem, Fredholm operators.

The first author gratefully acknowledges the financial support of the DAAD (Deutscher Akademischer Austauschdienst) und the Russian Ministry of Education, No 1.719.2016/2.2, and the grant of the Russian Federation Government for scientific research under the supervision of leading scientist at the Siberian Federal University, contract No 14.Y26.31.0006. 
10. The Fischer-Riesz equations 24

$\begin{array}{lll}11 . & \text { Regularisation of solutions } & 27\end{array}$

$\begin{array}{lll}12 . & \text { Solvability of elliptic boundary value problems } & 29\end{array}$

References 30

\section{INTRODUCTION}

Boundary value problems for first order elliptic systems is a classical area of analysis which goes back as far as Hilbert and Poincaré, see Gak77. As but one of the central topics in the $1960 \mathrm{~s}$ we mention the study of those first order elliptic systems which possess boundary conditions with the Shapiro-Lopatinskij property. The purely algebraic Shapiro-Lopatinskij condition proves actually to be equaivalent to the Fredholm property of the boundary value problem in classical Sobolev spaces.

The Fredholm property just amounts to saying that the null-space of the problem is of finite dimension and the problem is solvable for a subspace of data $\left(f, u_{0}\right)$ having finite codimension. Hence, for the existence of a solution it is necessary and sufficient that the data satisfy a finite number of moment conditions, i.e., those of the form $F\left(f, u_{0}\right)=0$, where $F$ is a continuous linear functional on the data space. Relevant continuous linear functionals on the data space are obtained from a Green formula which is used to introduce the so-called adjoint boundary value problem with respect to the Green formula. The Green formula actually establishes a duality on manifolds with boundary which suits well to the setting of a boundary value problem.

The adjoint boundary value problem need not satisfy the Shapiro-Lopatinskij condition even if the original problem does. Sometimes this is formulated inappropriately by saying that there is no adjoint boundary value problem, see for instance Ste93b. In fact, the paper Ste93b shows that a Hilbert problem for the MoisilTeodorescu system has no adjoint problem which bears the Shapiro-Lopatinskij property.

More precisely, let $\mathcal{X}$ be a bounded domain with smooth boundary in $\mathbb{R}^{3}$. In the source-free stationary case the field equations are

$$
\begin{aligned}
\operatorname{div} u & =0, \\
\operatorname{curl} u & =0
\end{aligned}
$$

in $\mathcal{X}$. We subject the vector field $u$ to the boundary condition

$$
b_{0} u^{1}+b_{1} u^{2}+b_{2} u^{3}=u_{0}
$$

on $\partial \mathcal{X}$, where $b=\left(b_{0}, b_{1}, b_{2}\right)$ is a given vector of length one and $u_{0}$ a prescribed function. This boundary value problem is embedded into a Riemann-Hilbert problem for the so-called Moisil-Teodorescu system

$$
\begin{aligned}
\operatorname{div} u & =0, \\
\operatorname{grad} u^{4}+\operatorname{curl} u & =0
\end{aligned}
$$

in $\mathcal{X}$ by introducing an additional unknown function $u^{4}$ satisfying $u^{4}=0$ on $\partial \mathcal{X}$. Since the latter system readily implies that $\Delta u^{4}=0$ in $\mathcal{X}$, it follows that $u^{4}$ vanishes identically in $\mathcal{X}$, and so we return to the original boundary value problem (0.1), 0.2 . 
Write $B$ for the $(2 \times 4)$-matrix whose rows are $(b, 0)$ and $(0,0,0,1)$. The rank of $B(x)$ just amounts to 2 for all $x \in \partial \mathcal{X}$. The Shapiro-Lopatinskij condition proves to be fulfilled. However, a purely topological argument shows that if $\mathcal{X}$ is homeomorphic to a ball then $B$ can not be framed in a regular $(4 \times 4)$-matrix of continuous functions on the entire boundary. In [Ste93b this is summarised by saying that the Riemann-Hilbert problem for the Moisil-Teodorescu system has no adjoint problem.

This paper begins with the observation that if one is allowed to frame $B$ in $(5 \times 4)$-matrices of maximal rank then the topological obstruction disappears. In this case we give an explicit construction for the adjoint boundary value problem. The construction is based on the compatibility complex for the overdetermined operator $B^{*}$ on the boundary. In fact it still works in much more general context of boundary value problems for first order systems provided that the rank of $B$ is maximal on $\partial \mathcal{X}$.

The Green formula obtained in this way establishes a splitting of the Cauchy data on $\partial \mathcal{X}$ of functions with values in $\mathbb{R}^{4}$ defined near the boundary. The Cauchy problem for the Moisil-Teodorescu system in $\mathcal{X}$ is normally solvable. This allows one to reduce the Riemann-Hilbert problem in $\mathcal{X}$ to a system of integral equations on the hypersurface $\partial \mathcal{X}$. We study this system by a boundary Fourier method, see [AT16]. The central point of this study is to explicitly construct a system of solutions to the formally adjoint system in a neighbourhood of $\overline{\mathcal{X}}$ which is complete in a subspace of $L^{2}\left(\partial \mathcal{X}, \mathbb{R}^{4}\right)$.

The approach of [AT13, AT16] does not work in the full generality. The aim of this paper is to develop the boundary Fourier method to derive solvability conditions and regularisation formulas for solutions of boundary value problems for first order elliptic systems.

\section{Part 1. The Riemann-Hilbert boundary value problem}

The theory of elliptic boundary value problems develops mostly about the Fredholm property which yields especially the solvability conditions. Since the presence of a boundary displays the singularity, the ellipticity of a boundary value problem amounts to the invertibility of two symbol mappings. The first of the two is the interior symbol and its invertibility means the ellipticity of the system of differential operators in $\mathcal{X}$. And the second one is the boundary symbol whose invertibility substitutes for the Shapiro-Lopatinskij condition. The verification of the Shapiro-Lopatinskij condition is by no means obvious. In this chapter we study the Riemann-Hilbert problem for solutions of a first order elliptic system. The main focus is on the boundary conditions which guarantee that the boundary value problem is Fredholm.

\section{Setting of the PRoblem}

Let $\mathcal{X}$ be a bounded domain with smooth boundary in $\mathbb{R}^{n+1}$. Consider a system of partial differential equations of the form

$$
A u:=\sum_{j=0}^{n} A_{j} \frac{\partial}{\partial x^{j}} u=f
$$


for an unknown function $u$ in $\mathcal{X}$ with values in $\mathbb{C}^{k}$. We assume that $A_{0}=E_{k}$ is the unit matrix of type $(k \times k)$ and $A_{i} A_{j}+A_{j} A_{i}=-2 \delta_{i j} E_{k}$ for all $i, j=1, \ldots, n$. Thus, (1.1) is a generalised Cauchy-Riemann system, cf. [Ste91, Ste93a, Ste93b. Moreover, 1.1) contains an inhomogeneous term $f$, a given function in $\mathcal{X}$ with values in $\mathbb{C}^{k}$.

Unless otherwise stated we assume that the $(k \times k)$-matrices $A_{j}$ are unitary. From the equalities $A_{j}^{-1}=-A_{j}$ it follows that in our case the unitarity just amounts to saying that

$$
A_{j}^{*}=-A_{j}
$$

for all $j=1, \ldots, n$. One looks for a solution $u$ of 1.1 which satisfies additionally the boundary condition

$$
B u=\left(B_{1}, B_{2}\right) u=u_{0}
$$

on $\partial \mathcal{X}$, where $B_{1}$ and $B_{2}$ are $(k / 2 \times k / 2)$-matrices of continuous complex-valued function on $\partial \mathcal{X}$. It is required that the rows of the $(k / 2 \times k)$-matrix $\left(B_{1}, B_{2}\right)$ are linearly independent at each boundary point. And $u_{0}$ is a continuous function with values in $\mathbb{C}^{k / 2}$.

\section{The MAin theOREM ON ELLIPTIC Boundary VALUe PROBlemS}

We go to recall the main result of the theory of elliptic boundary problems in bounded domains with smooth boundary which we use in the work (see for instance Vol65).

Let $\mathcal{X}$ be a bounded domain with smooth boundary in $\mathbb{R}^{n+1}$. Consider an inhomogeneous system of partial differential equations

$$
A u(x):=\sum_{|\alpha| \leq m} A_{\alpha}(x) \partial^{\alpha} u(x)=f(x)
$$

for an unknown function $u$ in $\mathcal{X}$ with values in $\mathbb{C}^{k}$. The coefficients $A_{\alpha}$ are assumed to be $(k \times k)$-matrices of smooth complex-valued functions in a neighbourhood of the closure of $\mathcal{X}$.

As usual, one defines the principal homogeneous symbol of the operator $A$ by the formula

$$
\sigma^{m}(A)(x, \xi)=\sum_{|\alpha|=m} A_{\alpha}(x)(\imath \xi)^{\alpha}
$$

for all $(x, \xi) \in \overline{\mathcal{X}} \times \mathbb{R}^{n+1}$. Given any fixed $(x, \xi)$, the matrix $\sigma^{m}(A)(x, \xi)$ is specified within the linear mappings of $\mathbb{C}^{k}$.

Example 2.1. The Cauchy-Riemann operator on the plane

$$
A u=\frac{1}{2}\left(\frac{\partial}{\partial x^{0}}+\imath \frac{\partial}{\partial x^{1}}\right) u
$$

has the principal homogeneous symbol $\sigma^{1}(A)(x, \xi)=(\imath / 2)\left(\xi_{0}+\imath \xi_{1}\right)$ defined for all $(x, \xi) \in \mathbb{R}^{2} \times \mathbb{R}^{2}$.

We want to formulate the condition of Shapiro-Lopatinskij at a point $x_{0} \in \partial \mathcal{X}$. Since the boundary of $\mathcal{X}$ is a smooth hypersurface in $\mathbb{R}^{n+1}$, one can choose local coordinates $x=\left(x^{0}, x^{1}, \ldots, x^{n}\right)$ in a neighbourhood of $x_{0}$ in $\mathbb{R}^{n+1}$ in such a way that the domain $\mathcal{X}$ is given by $\left\{x^{0}>0\right\}$ close to $x_{0}$. Then the unit vector of the $x^{0}$-axis is the inward normal vector of the hypersurface $\partial \mathcal{X}$ at $x_{0}$. Set $t=x^{0}$ and write $y=\left(x^{1}, \ldots, x^{n}\right)$ for the local coordinates of the boundary nearby $x_{0}$. Of 
course, the equation $A u=f$ changes in the new coordinates but we still keep the same designation for it. If one freezes the coefficients of $A$ at the point $x_{0}$ and applies the Fourier transform

$$
F_{y \mapsto \eta} f:=\int_{\mathbb{R}^{n}} \exp (-\imath\langle\eta, y\rangle) f(y, \cdot) d y
$$

in the tangential variables $y$ to the equation $A u=f$, then one obtains

$$
\sum_{\left|\beta+e_{j}\right| \leq m} A_{\beta+e_{j}}\left(x_{0}\right)(\imath \eta)^{\beta} \partial_{t}^{j} F_{y \mapsto \eta} u=F_{y \mapsto \eta} f
$$

for $t>0$ and all $\eta \in \mathbb{R}^{n}$. For any fixed $\eta \neq 0$, we now consider the initial value problem

$$
\begin{aligned}
\sigma^{m}(A)\left(x_{0}, \eta, \frac{1}{\imath} \frac{d}{d t}\right) v(t) & =0, \quad \text { if } t>0, \\
\sigma^{m_{j}}\left(B_{j}\right)\left(x_{0}, \eta, \frac{1}{\imath} \frac{d}{d t}\right) v(t) & =0, \quad \text { if } t=0,
\end{aligned}
$$

on the half-axis $t>0$, where $B_{j}$ are certain scalar partial differential operators close to the boundary of $\mathcal{X}$, for $j=0,1, \ldots,[m k / 2]-1$. The Shapiro-Lopatinskij condition reads as follows: For each $\eta \in T_{x_{0}}^{*}(\partial \mathcal{X})$ the initial value problem 2.1) posseses precisely one solution $v=v(t)$ which behaves sufficiently "well" at infinity, namely $v(t)=o(1)$.

A boundary value problem $\mathcal{A}=\{A, B\}$ with $B=\left\{B_{j}\right\}$ is said to be elliptic if $A$ is an elliptic operator in $\overline{\mathcal{X}}$ and the Shapiro-Lopatinskij condition is fulfilled at each point $x_{0} \in \partial \mathcal{X}$.

Let $V$ and $W$ be Banach spaces. We call a bounded linear operator $\mathcal{A}: V \rightarrow W$ Fredholm if the null space of $\mathcal{A}$ is finite dimensional, the range of $\mathcal{A}$ is closed and has a finite codimension, i.e., $\operatorname{dim} W / \operatorname{im} \mathcal{A}<\infty$. The latter property implies immediately that if $\mathcal{A}$ is Fredholm then for the inhomogeneous equation $\mathcal{A} v=w$ to have a solution it is necessary and sufficient that $w$ would satisfy a finite number of moment conditions.

Each boundary value problem

$$
\begin{cases}A u(x)=f(x), & \text { if } \quad x \in \mathcal{X} \\ B u(x)=u_{0}(x), & \text { if } \quad x \in \partial \mathcal{X}\end{cases}
$$

gives rise to a bounded linear operator

$$
\mathcal{A}: H^{s}\left(\mathcal{X}, \mathbb{C}^{k}\right) \rightarrow \underset{\oplus H^{s-m_{j}-1 / 2}(\partial \mathcal{X})}{\oplus}
$$

where $s$ is a natural number satisfying $s \geq m$. Here, $H^{s}\left(\mathcal{X}, \mathbb{C}^{k}\right)$ stands for the scale of Sobolev spaces of functions on $\mathcal{X}$ with values in $\mathbb{C}^{k}$ based on $L^{2}(\mathcal{X})$, the index $s$ running over the nonnegative integer numbers and even over all real numbers in a familiar way.

Theorem 2.2. Suppose $\mathcal{X}$ is a bounded domain in $\mathbb{R}^{n+1}$ with boundary of class $C^{s}$. The following are equivalent:

1) The boundary value problem of (2.2) is elliptic.

2) The operator $\mathcal{A}$ is Fredholm.

3) For all $u \in H^{s}\left(\mathcal{X}, \mathbb{C}^{k}\right)$ the a priori estimate

$$
\|u\|_{H^{s}\left(\mathcal{X}, \mathbb{C}^{k}\right)} \leq c\left(\|A u\|_{H^{s-m}\left(\mathcal{X}, \mathbb{C}^{k}\right)}+\|B u\|_{\oplus H^{s-m_{j}-1 / 2}(\partial \mathcal{X})}+\|u\|_{L^{2}\left(\mathcal{X}, \mathbb{C}^{k}\right)}\right)
$$


is fulfilled with $c$ a constant independent of $u$.

Proof. See, e.g., Agr97.

As but one consequence of Theorem 2.2 we mention a local regularity theorem for solutions of $(2.2)$ in Sobolev spaces.

\section{Applications to the Riemann-Hilbert problem}

As mentioned the Fredholm property is of great importance in the study of solvability of linear equations. The question arising is now under what algebraic conditions on $\{A, B\}$ the boundary value problem of $(2.2)$ is elliptic. Before we turn towards it let us recall that the canonical scalar product in $\mathbb{C}^{k}$ is given by the formula $(u, v):=v^{*} u$ for $u, v \in \mathbb{C}^{k}$, where $v^{*}=\bar{v}^{T}$ means the adjoint line for the column $v$.

Let $\mathcal{X} \subset \mathbb{R}^{n+1}$ is a bounded domain with boundary of class $C^{s}$ with $s \geq 1$. We denote by $\nu(x)=\left(\nu_{0}(x), \nu_{1}(x), \ldots, \nu_{n}(x)\right)$ the inward unit normal vector for $\partial \mathcal{X}$ at a point $x \in \partial \mathcal{X}$. Consider the Riemann-Hilbert boundary value problem (1.1), 1.2 with unitary matrices $A_{j}$. It is required that the entries of $B_{1}, B_{2}$ are $C^{s}$ functions on $\partial \mathcal{X}$. Without restriction of generality one can assume that the rows of the $(k / 2 \times k)$-matrix $B=\left(B_{1}, B_{2}\right)$ are orthonormal. For if not, we apply the Gram-Schmidt orthogonalisation process to the rows of $B$, thus obtaining an equivalent boundary condition.

Theorem 3.1. The boundary value problem (1.1), (2.2) is Fredholm in Sobolev spaces $u \in H^{s}\left(\mathcal{X}, \mathbb{C}^{k}\right)$ and $f \in H^{s-1}\left(\mathcal{X}, \mathbb{C}^{k}\right), u_{0} \in H^{s-1 / 2}\left(\partial \mathcal{X}, \mathbb{C}^{k / 2}\right)$ if and only if

$$
\operatorname{det}\left(B\left(\sum_{i=0}^{n} \nu_{i}(x) A_{i}^{*}\right)\left(\sum_{j=0}^{n} \tau_{j}(x) A_{j}\right) B^{*}-\imath E_{k / 2}\right) \neq 0
$$

for all $x \in \partial \mathcal{X}$ and all tangent unit vectors $\tau(x)=\left(\tau_{0}(x), \tau_{1}(x), \ldots, \tau_{n}(x)\right)$ to $\partial \mathcal{X}$ at $x$.

In order to prove the theorem we need some preparation. Given any $(k \times k)$ matrix $T$ of complex numbers, a vector $v \neq 0$ is called an eigenvector of $T$ to an eigenvalue $\lambda$ if $T v=\lambda v$, i.e., if $\left(T-\lambda E_{k}\right) v=0$. The eiqenvalues of the matrix $T$ are actually solutions of the equation $\sin \operatorname{det}\left(T-\lambda E_{k}\right)=0$. As is known, to each eigenvalue $\lambda$ of $T$ there is precisely $k-\operatorname{rank}\left(T-\lambda E_{k}\right)$ linearly independent eigenvectors.

Lemma 3.2. Suppose $A_{1}, \ldots, A_{n}$ are $(k \times k)$-matrices of complex numbers satisfying $A_{i} A_{j}+A_{j} A_{i}=-2 \delta_{i j} E_{k}$ for $i, j=1, \ldots, n$. For each $\xi=\left(\xi_{1}, \ldots, \xi_{n}\right) \in \mathbb{R}^{n} \backslash\{0\}$, the matrix

$$
\imath \sum_{j=1}^{n} \xi_{j} A_{j}
$$

has precisely two eigenvalues $\lambda= \pm|\xi|$, and all eigenvectors to these eigenvalues have the form

$$
v=\left( \pm|\xi| E_{k}+\imath \sum_{j=1}^{n} \xi_{j} A_{j}\right) c
$$

woth $c \in \mathbb{C}^{k}$. If $c$ runs over all vectors in $\mathbb{C}^{k}$, then $v$ is either an eigenvector or the null vector. Moreover, to each eigenvalue there are precisely $k / 2$ linearly independent eigenvectors. 
Proof. Let

$$
\operatorname{det}\left(\imath \sum_{j=1}^{n} \xi_{j} A_{j}-\lambda E_{k}\right)=0
$$

From

$$
\left(\imath \sum_{j=1}^{n} \xi_{j} A_{j}-\lambda E_{k}\right)\left(\imath \sum_{k=1}^{n} \xi_{k} A_{k}+\lambda E_{k}\right)=\left(|\xi|^{2}-\lambda^{2}\right) E_{k}
$$

it follows that $|\xi|^{2}-\lambda^{2}=0$, and so $\lambda= \pm|\xi|$. Obviously, $\lambda= \pm|\xi|$ satisfies equation $(3.2)$. We now show that

$$
\operatorname{rank}\left(\imath \sum_{j=1}^{n} \xi_{j} A_{j} \pm|\xi| E_{k}\right) \geq \frac{k}{2}
$$

Every matrix $A_{j}$ can be reduced by means of certain equivalence transformation to a block matrix. In this way the matrices

$$
\imath \sum_{j=1}^{n} \xi_{j} A_{j} \pm|\xi| E_{k}
$$

are equivalent to matrices of block structure, each block being of the form

$$
\left(\begin{array}{cc} 
\pm|\xi| E_{2^{m-1}}+\imath \sum_{j=3}^{n} \xi_{j} A_{j-2}^{\prime} & -\left(\xi_{1}+\imath \xi_{2}\right) E_{2^{m-1}} \\
-\left(\xi_{1}-\imath \xi_{2}\right) E_{2^{m-1}} & \pm|\xi| E_{2^{m-1}}-\imath \sum_{j=3}^{n} \xi_{j} A_{j-2}^{\prime}
\end{array}\right)
$$

and of the type $2^{m} \times 2^{m}$ with $n=2 m$ or $n=2 m+1$, where $k$ is a multiple of $2^{m}$, (see $[$ Ste93b] $)$. It is clear that the $\left(2^{m-1} \times 2^{m-1}\right)$-matrices $-\left(\xi_{1} \pm \imath \xi_{2}\right) E_{2^{m-1}}$ have maximal rank, if $\xi_{1}^{2}+\xi_{2}^{2} \neq 0$. If $\xi_{1}=\xi_{2}=0$, then we have to show that the other two submatrices

$$
\pm|\xi| E_{2^{m-1}} \pm \imath \sum_{j=3}^{n} \xi_{j} A_{j-2}^{\prime}
$$

are of rank $\geq 2^{m-2}$. We verify this fact only for the submatrix in the left upper corner.

On using the block structure of matrices in question we get once again

$$
\pm|\xi| E_{2^{m-1}}+\imath \sum_{j=3}^{n} \xi_{j} A_{j-2}^{\prime}=\left(\begin{array}{cc} 
\pm|\xi| E_{2^{m-2}}+\imath \sum_{j=5}^{n} \xi_{j} A_{j-4}^{\prime \prime} & -\left(\xi_{3}+\imath \xi_{4}\right) E_{2^{m-2}} \\
-\left(\xi_{3}-\imath \xi_{4}\right) E_{2^{m-2}} & \pm|\xi| E_{2^{m-2}}-\imath \sum_{j=5}^{n} \xi_{j} A_{j-4}^{\prime \prime}
\end{array}\right) .
$$

If $\xi_{3}^{2}+\xi_{4}^{2} \neq 0$, then the rank of this matrix is clearly $\geq 2^{m-2}$. If, however, both $\xi_{3}$ and $\xi_{4}$ vanish, then we split the remaining submatrices and show that they have rank $\geq 2^{k-3}$.

We continue in this fashion obtaining either a pair of numbers $\left(\xi_{2 j-1}, \xi_{2 j}\right)$ satisfying $\xi_{2 j-1}^{2}+\xi_{2 j}^{2} \neq 0$, in which case the lemma is obvious. Or we finally get a $(2 \times 2)$-matrix of the form

$$
\left(\begin{array}{cc} 
\pm|\xi| & -\xi_{n-1}-\imath \xi_{n} \\
-\xi_{n-1}+\imath \xi_{n} & \pm|\xi|
\end{array}\right)
$$


if $n$ is even, and

$$
\left(\begin{array}{cc} 
\pm|\xi|-\xi_{n} & -\xi_{n-2}-\imath \xi_{n-1} \\
-\xi_{n-2}+\imath \xi_{n-1} & \pm|\xi|+\xi_{n}
\end{array}\right)
$$

if $n$ is odd. The ranks of both matrices are 1 . We have thus proved that the block matrices of the type $2^{m} \times 2^{m}$ have rank $\geq 2^{m-1}$. Hence, the entire matrix has rank $\geq k / 2$. Since

$$
\left(\imath \sum_{j=1}^{n} \xi_{j} A_{j} \mp|\xi| E_{k}\right)\left(\imath \sum_{j=1}^{n} \xi_{j} A_{j} \pm|\xi| E_{k}\right) c=0
$$

for all $c \in \mathbb{C}^{k}$, the vectors

$$
\left(\imath \sum_{j=1}^{n} \xi_{j} A_{j} \pm|\xi| E_{k}\right) c \neq 0
$$

are eigenvectors to the eigenvalues $\lambda= \pm|\xi|$. Since the number of linearly independent eigenvectors does not exceed $k$, the rank of any matrix

$$
\imath \sum_{j=1}^{n} \xi_{j} A_{j} \pm|\xi| E_{k}
$$

just amounts to $k / 2$. Besides, there is no other eigenvectors different from those which we have already given.

We now turn to the proof of Theorem 3.1 .

Proof. By the main theorem of elliptic boundary value problems, the Fredholm property is equivalent to the ellipticity of the problem. The ellipticity of generalised Cauchy-Riemann equations is a routine fact. Hence, it remains to show that relation (3.1) is equivalent to the Shapiro-Lopatinskij condition.

To this end pick an arbitrary boundary point $x_{0} \in \partial \mathcal{X}$. We choose a local coordinate system $X^{0}, X^{1}, \ldots, X^{n}$ with the property that the origin is at $x_{0}$, the $X^{0}$-axis coincides with the inward normal to $\partial \mathcal{X}$ at $x_{0}$, the $X^{1}, \ldots, X^{n}$-axes lie in the tangential hyperplane of $\partial \mathcal{X}$ at $x_{0}$, and the system $X^{0}, X^{1}, \ldots, X^{n}$ is obtained from the global coordinate system $x^{0}, x^{1}, \ldots, x^{n}$ by means of translation and rotation, i.e.,

$$
\begin{aligned}
x^{i} & =\sum_{j=0}^{n} u_{j}^{i} X^{j}+x_{0}^{i}, \\
X^{j} & =\sum_{j=0}^{n} u_{i}^{j}\left(x^{i}-x_{0}^{i}\right)
\end{aligned}
$$

with an appropriate orthogonal matrix $U=\left(u_{j}^{i}\right)_{\substack{i=0,1, \ldots, n \\ j=0,1, \ldots, n}}$.

Hence it follows that

$$
\frac{\partial}{\partial x^{i}}=\sum_{j=0}^{n} u_{j}^{i} \frac{\partial}{\partial X^{j}}
$$


and

$$
\begin{aligned}
\sum_{i=0}^{n} A_{i} \frac{\partial}{\partial x^{i}} u & =\sum_{i=0}^{n} A_{i}\left(\sum_{j=0}^{n} u_{j}^{i} \frac{\partial}{\partial X^{j}} u\right) \\
& =\sum_{j=0}^{n}\left(\sum_{i=0}^{n} u_{j}^{i} A_{i}\right) \frac{\partial}{\partial X^{j}} u .
\end{aligned}
$$

By Ste91, the generalised Cauchy-Riemann equations are rotation invariant. Therefore, in the coordinates $X^{0}, X^{1}, \ldots, X^{n}$ we get a new generalised Cauchy-Riemann system

$$
\sum_{j=0}^{n} C_{j} \frac{\partial}{\partial X^{j}} u=\left(\sum_{i=0}^{n} u_{0}^{i} A_{i}^{-1}\right) f
$$

where

$$
C_{k}=\left(\sum_{i=0}^{n} u_{0}^{i} A_{i}^{-1}\right)\left(\sum_{j=0}^{n} u_{k}^{j} A_{j}\right) .
$$

The matrices $C_{k}$ are unitary, i.e., $C_{k}^{*}=-C_{k}$ for $k=1, \ldots, n$.

We set $X^{0}=t$ and apply the Fourier transform in the variables $X^{1}, \ldots, X^{n}$ to the principal part of the generalised Cauchy-Riemann system. In this way we arrive at the homogeneous system of ordinary differential equations

$$
\frac{d}{d t} v(t)+\imath \sum_{j=1}^{n} \xi_{j} C_{j} v(t)=0
$$

on the semiaxis $t \geq 0$. We now freeze the coefficients of the boundary conditions at the origin of the local coordinate system. On applying the Fourier transform to the boundary condition one obtains

$$
\left(B_{1}, B_{2}\right) v(0)=0 \text {, }
$$

where $B_{i}=B_{i}(0)$ for $i=1,2$. The Shapiro-Lopatinskij condition at the point $x_{0} \in \partial \mathcal{X}$ just amounts to saying that for all $\xi=\left(\xi_{1}, \ldots, \xi_{n}\right) \in \mathbb{R}^{n} \backslash\{0\}$ the initial value problem possesses only the trivial solution in the space of stable solutions on $[0, \infty)$.

We substitute

$$
v(t)=\exp (\lambda t) c
$$

in the system and obtain

$$
\left(\lambda E_{k}+\imath \sum_{j=1}^{n} \xi_{j} C_{j}\right) c=0 .
$$

By Lemma 3.2 there are precisely two eigenvalues $\lambda= \pm|\xi|$. We get stable solutions merely for $\lambda=-|\xi|$ and the corresponding $k / 2$ linearly independent eigenvectors are of the form

$$
c=\left(|\xi| E_{k}+\imath \sum_{j=1}^{n} \xi_{j} C_{j}\right) c^{\prime},
$$

where $c^{\prime} \in \mathbb{C}^{k}$. Hence it follows that any stable solution to the sytem on $[0, \infty)$ is of the form

$$
v(t)=\exp (-|\xi| t)\left(|\xi| E_{k}+\imath \sum_{j=1}^{n} \xi_{j} C_{j}\right) c^{\prime}
$$


with $c^{\prime} \in \mathbb{C}^{k}$.

Since the matrices $C_{j}$ are unitary, the matrix

$$
\imath \sum_{j=1}^{n} \xi_{j} C_{j}
$$

is Hermitean. Therefore, it can be reduced by means of a unitary matrix $T$ to a diagonal form, $T$ depending on $\xi$ and $U=\left(u_{j}^{i}\right)$. We thus conclude that

$$
\imath \sum_{j=1}^{n} \xi_{j} C_{j}=|\xi| T\left(\begin{array}{cc}
E_{k / 2} & 0 \\
0 & -E_{k / 2}
\end{array}\right) T^{*}
$$

whence

$$
\begin{aligned}
v(t) & =\exp (-|\xi| t)\left(|\xi| E_{k}+|\xi| T\left(\begin{array}{cc}
E_{k / 2} & 0 \\
0 & -E_{k / 2}
\end{array}\right) T^{*}\right) c^{\prime} \\
& =2|\xi| \exp (-|\xi| t) T\left(\begin{array}{cc}
E_{k / 2} & 0 \\
0 & 0
\end{array}\right) T^{*} c^{\prime} .
\end{aligned}
$$

Write

$$
T=\left(\begin{array}{cc}
T_{1}^{1} & T_{2}^{1} \\
T_{1}^{2} & T_{2}^{2}
\end{array}\right)
$$

where $T_{j}^{i}$ are $(k / 2 \times k / 2)$-matrices, and

$$
T^{*} c^{\prime}=\left(\begin{array}{c}
z \\
w
\end{array}\right)
$$

where $z, w \in \mathbb{C}^{k / 2}$. Then

$$
v(t)=2|\xi| \exp (-|\xi| t)\left(\begin{array}{c}
T_{1}^{1} \\
T_{1}^{2}
\end{array}\right) z
$$

is a stable solution for arbitrary $z \in \mathbb{C}^{k / 2}$.

We substitute this formula into the initial value problem to obtain

$$
\left(B_{1}, B_{2}\right) v(0)=|\xi|\left(B_{1}, B_{2}\right)\left(\begin{array}{c}
T_{1}^{1} \\
T_{1}^{2}
\end{array}\right) z=0 .
$$

Hence, $v(t)=0$ if and only if $z=0$. And the initial value problem possesses only the trivial solution if and only if

$$
\operatorname{det}\left(\left(B_{1}, B_{2}\right)\left(\begin{array}{c}
T_{1}^{1} \\
T_{1}^{2}
\end{array}\right)\right) \neq 0
$$

We set

$$
\left(Q_{1}, Q_{2}\right):=\left(B_{1}, B_{2}\right) T
$$

where $Q_{1}, Q_{2}$ are matrices of type $k / 2 \times k / 2$. Equality $(3.3)$ means that

$$
\operatorname{det} Q_{1} \neq 0 \text {. }
$$

Since $\operatorname{det} Q_{1}^{*}=\overline{\operatorname{det} Q_{1}}$, the relation $\operatorname{det} Q_{1} \neq 0$ is equivalent to $\operatorname{det} Q_{1} Q_{1}^{*} \neq 0$. Using the equality

$$
Q_{1} Q_{1}^{*}+Q_{2} Q_{2}^{*}=E_{k / 2}
$$


we get

$$
\begin{aligned}
2 Q_{1} Q_{1}^{*} & =\left(Q_{1}, Q_{2}\right)\left(\begin{array}{cc}
E_{k / 2} & 0 \\
0 & -E_{k / 2}
\end{array}\right)\left(\begin{array}{c}
Q_{1}^{*} \\
Q_{2}^{*}
\end{array}\right)+E_{k / 2} \\
& =\left(B_{1}, B_{2}\right) T\left(\begin{array}{cc}
E_{k / 2} & 0 \\
0 & -E_{k / 2}
\end{array}\right) T^{*}\left(\begin{array}{c}
B_{1}^{*} \\
B_{2}^{*}
\end{array}\right)+E_{k / 2} \\
& =\left(B_{1}, B_{2}\right) \frac{\imath}{|\xi|} \sum_{j=1}^{n} \xi_{j} C_{j}\left(\begin{array}{c}
B_{1}^{*} \\
B_{2}^{*}
\end{array}\right)+E_{k / 2} \\
& =\imath\left(\left(B_{1}, B_{2}\right) \sum_{j=1}^{n} \frac{\xi_{j}}{|\xi|} C_{j}\left(\begin{array}{c}
B_{1}^{*} \\
B_{2}^{*}
\end{array}\right)-\imath E_{k / 2}\right) .
\end{aligned}
$$

As already mentioned,

$$
C_{k}=\left(\sum_{i=0}^{n} u_{0}^{i} A_{i}^{-1}\right)\left(\sum_{j=0}^{n} u_{k}^{j} A_{j}\right)
$$

whence

$$
\sum_{k=1}^{n} \frac{\xi_{k}}{|\xi|} C_{k}=\left(\sum_{i=0}^{n} u_{0}^{i} A_{i}^{-1}\right)\left(\sum_{\substack{j=0,1, \ldots, n \\ k=1, \ldots, n}} \frac{\xi_{k}}{|\xi|} u_{k}^{j} A_{j}\right)
$$

Obviously, the vector $\left(u_{0}^{i}\right)_{i=0,1, \ldots, n}$ presents the inward normal vector $\nu$ and the vector

$$
\left(\sum_{j=1}^{n} \frac{\xi_{j}}{|\xi|} u_{j}^{i}\right)_{i=0,1, \ldots, n}
$$

is a tangential vector $\tau$ of length 1 at the boundary. Hence we get

$$
\sum_{k=1}^{n} \frac{\xi_{k}}{|\xi|} C_{k}=\left(\sum_{i=0}^{n} \nu_{i} A_{i}^{*}\right)\left(\sum_{j=0}^{n} \tau_{j} A_{j}\right) .
$$

On substituting this formula into 3.4 and taking into account that $\operatorname{det} Q_{1} Q_{1}^{*} \neq 0$ we obtain

$$
\begin{aligned}
\operatorname{det}\left(-2 \imath Q_{1} Q_{1}^{*}\right) & =\operatorname{det}\left(\left(B_{1}, B_{2}\right)\left(\sum_{j=0}^{n} \nu_{j} A_{j}^{*}\right)\left(\sum_{j=0}^{n} \tau_{j} A_{j}\right)\left(\begin{array}{c}
B_{1}^{*} \\
B_{2}^{*}
\end{array}\right)-\imath E_{k / 2}\right) \\
& \neq 0,
\end{aligned}
$$

as desired.

\section{Part 2. The Moisil-Teodoresco system}

In this chapter we apply elliptic theory to a Riemann-Hilbert problem for the Moisil-Teodoresco system. For this purpose we construct an explicit Green formula which is of great importance to present the (formal) adjoint boundary value problem. 


\section{The Green formula}

Consider the boundary value problem

$$
\begin{aligned}
A u:=\sum_{j=0}^{n} A_{j} \partial_{j} u & =f \quad \text { in } \mathcal{X}, \\
B u & =u_{0} \text { on } \partial \mathcal{X},
\end{aligned}
$$

where we assume without restriction of generality that the matrices $A_{j}$ are unitary and $B$ is a matrix of continuous functions on $\partial \mathcal{X}$ whose rank is maximal at each point of the boundary.

Lemma 4.1. For all $u, g \in C^{1}\left(\overline{\mathcal{X}}, \mathbb{C}^{k}\right)$ it follows that

$$
\int_{\partial \mathcal{X}} g^{*} \sigma^{1}(A)(\imath \nu) u d s=\int_{\mathcal{X}}\left(g^{*} A u-\left(A^{*} g\right)^{*} u\right) d x,
$$

where $\nu$ is the inward unit normal vector for $\partial \mathcal{X}$ and ds the surface measure on the boundary $\partial \mathcal{X}$.

As usual, $A^{*}$ stands for the formal adjoint of the differential operator $A$ acting on functions with values in $\mathbb{C}^{k}$. If one uses the canonical scalar product in $\mathbb{C}^{k}$, then the integrands of the boundary and spatial integrals can be written as $\left(\sigma^{1}(A)(\imath \nu) u, g\right)_{x}$ and $(A u, g)_{x}-\left(u, A^{*} g\right)_{x}$, respectively, where the sub $x$ points out the pointwise scalar product of function values.

Proof. Using the Gauß formula yields

$$
\begin{aligned}
\int_{\partial \mathcal{X}} g^{*} \sigma^{1}(A)(\imath \nu) u d s & =-\sum_{j=0}^{n} \int_{\partial \mathcal{X}} g^{*} A_{j} \nu_{j} u d s \\
& =\int_{\mathcal{X}} \sum_{j=0}^{n} \frac{\partial}{\partial x_{j}}\left(g^{*} A_{j} u\right) d x \\
& =\int_{\mathcal{X}}\left(\sum_{j=0}^{n}\left(\frac{\partial g}{\partial x_{j}}\right)^{*} A_{j} u+\sum_{j=0}^{n} g^{*} A_{j} \frac{\partial u}{\partial x_{j}}\right) d x \\
& =\int_{\mathcal{X}}\left(-\left(A^{*} g\right)^{*} u+g^{*} A u\right) d x
\end{aligned}
$$

as desired.

Let $B(x)$ be an arbitrary $(l \times k)$-matrix of continuous functions on the boundary with the property that $l \leq k$ and the rank of $B(x)$ is equal to $l$ for all $x \in \partial \mathcal{X}$ We set $l_{0}=l, l_{1}=k$ and choose an $\left(l_{2} \times k\right)$-matrix $C(x)$ of continuous functions on $\partial \mathcal{X}$, such that

$$
\operatorname{rank}\left(\begin{array}{c}
B(x) \\
C(x)
\end{array}\right)=k
$$

for all $x \in \partial \mathcal{X}$. Hence it follows that the $(k \times k)$-matrix

$$
T(x):=\left(\begin{array}{c}
B(x) \\
C(x)
\end{array}\right)^{*}\left(\begin{array}{c}
B(x) \\
C(x)
\end{array}\right)=B(x)^{*} B(x)+C(x)^{*} C(x)
$$

is invertible and the entries of the inverse matrix $T^{-1}(x)$ are continuous functions on the boundary. Actually, the matrix $C(x)$ can be chosen in such a way that $C(x) B(x)^{*}=0$ for all $x \in \partial \mathcal{X}$. This can be clarified within the framework of 
compatibility complexes for sufficiently regular differential operators. To wit, we start with $C^{0}=B^{*}$.

Lemma 4.2. Suppose that $C^{0}(x)$ is an $\left(l_{1} \times l_{0}\right)$-matrix of continuous functions on the boundary whose rank is $l_{0}$ for all $x \in \partial \mathcal{X}$. Then there are $\left(l_{i+1} \times l_{i}\right)$-matrices $C^{i}(x)$ of continuous functions on the boundary, with $i=1, \ldots, N-1$, such that the sequence

$$
0 \longrightarrow \mathbb{C}^{l_{0}} \stackrel{C^{0}(x)}{\longrightarrow} \mathbb{C}^{l_{1}} \stackrel{C^{1}(x)}{\longrightarrow} \ldots \stackrel{C^{N-1}(x)}{\longrightarrow} \mathbb{C}^{l_{N}} \longrightarrow 0
$$

is exact for each $x \in \partial \mathcal{X}$.

Proof. We focus on the construction of the matrix $C^{1}(x)$ which is used in the sequel. To this end, we start with any matrix $C(x)$ satisfying 4.2 , and modify it in such a way that $C(x) B(x)^{*}=0$ for all $x \in \partial \mathcal{X}$. This latter condition just amounts to saying that $C(x)$ vanishes on the range of $B(x)^{*}$. For each fixed $x \in \partial \mathcal{X}$, the space $\mathbb{C}^{k}$ decomposes into the orthogonal sum of the null space of $B(x)$ and the range of $B(x)^{*}$. Hence, we shall have got the desired modification of $C(x)$ if we compose $C(x)$ with the projection $P(x)$ of $\mathbb{C}^{k}$ onto the null space of $B(x)$. What is left is to show that there is any projection $P(x)$ which depends continuously on $x \in \partial \mathcal{X}$. Since the matrix $B(x)$ has rank $l$, it follows that the $(l \times l)$-matrix $B(x) B(x)^{*}$ is invertible for all $x \in \partial \mathcal{X}$ and the entries of the inverse are continuous functions on the boundary. Define

$$
P(x)=E_{k}-B(x)^{*}\left(B(x) B(x)^{*}\right)^{-1} B(x)
$$

for $x \in \partial \mathcal{X}$. Obviously, $P(x)$ is the identity operator on the null space of $B(x)$ and $B(x) P(x)=B(x)-B(x)=0$, i.e., $P(x)$ is a projection onto the null space of $B(x)$ indeed. On the other hand, $P(x)$ vanishes on the range of $B(x)^{*}$, for $P(x) B(x)^{*}=B(x)^{*}-B(x)^{*}=0$. Hence, on substituting $C(x) P(x)$ for $C(x)$ we obtain an $\left(l_{2} \times k\right)$-matrix $C^{1}(x)$ with desired properties.

We can thus choose $C(x)=C^{1}(x)$ to obtain $C B^{*}=0$ in 4.2 . Note that $T(x)$ just amounts to the Laplacian of the complex at step $\mathbb{C}^{l_{1}}$. No attempt has been made here to develop the theory for pseudodifferential operators $B$ on the boundary $\partial \mathcal{X}$.

Theorem 4.3. For all $u, g \in C^{1}\left(\overline{\mathcal{X}}, \mathbb{C}^{k}\right)$ it follows that

$$
\int_{\partial \mathcal{X}}\left(\left(C^{\text {adj }} g\right)^{*} B u-\left(B^{\text {adj }} g\right)^{*} C u\right) d s=\int_{\mathcal{X}}\left(g^{*} A u-\left(A^{*} g\right)^{*} u\right) d x,
$$

where

$$
\begin{aligned}
C^{\text {adj }}= & B T^{-1}\left(\sigma^{1}(A)(\imath \nu)\right)^{*}, \\
B^{\text {adj }}= & -C T^{-1}\left(\sigma^{1}(A)(\imath \nu)\right)^{*} .
\end{aligned}
$$

Proof. By the above, $T^{-1}(x) T(x)=E_{k}$ for all $x \in \partial \mathcal{X}$. On substituting the formula for $T(x)$ one obtains

$$
\left(T^{-1}(x) B(x)^{*}\right) B(x)+\left(T^{-1}(x) C(x)^{*}\right) C(x)=E_{k} .
$$

We now substitute this decomposition into the formula of Lemma 4.1. Then the boundary integral reduces to

$$
\begin{aligned}
& \int_{\partial \mathcal{X}} g^{*} \sigma^{1}(A)(\imath \nu) E_{k} u d s=\int_{\partial \mathcal{X}} g^{*} \sigma^{1}(A)(\imath \nu)\left(\left(T^{-1} B^{*}\right) B u+\left(T^{-1} C^{*}\right) C u\right) d s \\
& =\int_{\partial \mathcal{X}}\left(\left(C^{\mathrm{adj}} g\right)^{*} B u-\left(B^{\mathrm{adj}} g\right)^{*} C u\right) d s,
\end{aligned}
$$


showing the theorem.

The formula of Theorem 4.3 is called the Green formula for the boundary value problem $\{A, B\}$ in the domain $\mathcal{X}$. It allows one to introduce an adjoint boundary value problem for $\{A, B\}$, this is given by $\left\{A^{*}, B^{\text {adj }}\right\}$. A condition for the existence of an elliptic adjoint boundary value problem is that the matrix $B(x)$ can be supplemented with a matrix $C(x)$ to a continuously invertible matrix on the boundary of $\mathcal{X}$. This is not always the case. Moreover, the adjoint boundary value problem is not uniquely determined, for the supplement $C(x)$ can be chosen in diverse fashions.

Theorem 4.3 yields immediately a necessary condition for the existence of a solution $u \in H^{1}\left(\mathcal{X}, \mathbb{C}^{k}\right)$ to problem 4 .1) with given $f$ and $u_{0}$. We call it a solvability condition of problem 4.1 for short.

Corollary 4.4. If problem (4.1) possesses a solution $u \in H^{1}\left(\mathcal{X}, \mathbb{C}^{k}\right)$ in $\mathcal{X}$ then the data $f$ and $u_{0}$ should satisfy

$$
\int_{\partial \mathcal{X}}\left(u_{0}, C^{\mathrm{adj}} g\right)_{x} d s=\int_{\mathcal{X}}(f, g)_{x} d x
$$

for all $g \in H^{1}\left(\mathcal{X}, \mathbb{C}^{k}\right)$ such that $A^{*} g=0$ in $\mathcal{X}$ and $B^{\text {adj }} g=0$ on $\partial \mathcal{X}$.

Proof. We first observe that Theorem 4.3 holds not only for smooth functions $u$ and $g$ with values in $\mathbb{C}^{k}$ on the closure of $\mathcal{X}$ but also for all $u, g \in H^{1}\left(\mathcal{X}, \mathbb{C}^{k}\right)$. To see this it suffices to approximate the functions $u$ and $g$ of Sobolev class by functions $u_{j}$ and $g_{j}$ which are $C^{\infty}$ in the closure of $\mathcal{X}$. On writing the Green formula for $u_{j}$ and $g_{j}$ and passing to the limit when $j \rightarrow \infty$ we get the Green formula forfunctions $u$ and $g$ of Sobolev class $H^{1}\left(\mathcal{X}, \mathbb{C}^{k}\right)$. Equality (4.4) readily follows from the Green formula.

Corollary 4.4 initiates immediately a boundary value problem for solutions of the adjoint system $A^{*} g=v$ in $\mathcal{X}$. To wit,

$$
\begin{aligned}
A^{*} g:=-\sum_{j=0}^{n} A_{j}^{*} \partial_{j} g & =v \quad \text { in } \mathcal{X}, \\
B^{\text {adj } g} & =g_{0} \text { on } \partial \mathcal{X},
\end{aligned}
$$

where $B^{\text {adj }}=-C T^{-1}\left(\sigma^{1}(A)(\imath \nu)\right)^{*}$. Problem 4.5 is called the adjoint boundary value problem for (4.1) with respect to the Green formula. We mention two extreme cases of Corollary 4.4. If ( $l=k$ and) $B(x)$ is invertible for all $x \in \partial \mathcal{X}$, then (4.1) is a Cauchy problem with data on the whole boundary. From $C B^{*}=0$ we deduce that $C=0$. Thus, the boundary operator $B^{\text {adj }}$ is zero and the adjoint problem 4.5 reduces to the inhomogeneous equation $A^{*} g=v$ in $\mathcal{X}$. Since $A^{*}$ is a generalised Cauchy-Riemann operator, the equation $A^{*} g=v$ has a solution $g \in H^{1}\left(\mathcal{X}, \mathbb{C}^{k}\right)$ for all data $v \in L^{2}\left(\mathcal{X}, \mathbb{C}^{k}\right)$, the space of solutions being of infinite dimension. Condition (4.4) is known to be not only necessary but also sufficient for the solvability of the Cauchy problem 4.1, see [Tar95, 10.1.3]. If $(l=0$ then) the matrix $B(x)$ is zero for all $x \in \partial \mathcal{X}$, then problem (4.1) reduces to the inhomogeneous equation $A u=f$ in $\mathcal{X}$. Once again it has a solution $u \in H^{1}\left(\mathcal{X}, \mathbb{C}^{k}\right)$ for all data $f \in L^{2}\left(\mathcal{X}, \mathbb{C}^{k}\right)$ while the null space of $A$ is infinite dimensional. By 4.2 , the matrix $C(x)$ should bear rank $k$ at each boundary point $x$. Hence, the adjoint 4.5 is a Cauchy problem for solutions of $A^{*} g=0$ in $\mathcal{X}$ with data on the entire boundary. This problem is normally solvable 
and possesses at most one solution, see [Tar95, 10.1.3]. Formally, the condition of Corollary 4.4 is necessary and sufficient for the solvability of (4.1). One may conjecture that 4.4 is a necessary and sufficient condition for the solvability of 4.1 each time when this problem is Fredholm. But we have not been able to prove this.

\section{A Calculation for the Moisil-Teodoresco system}

In this section we present explicit calculations concerning a Riemann-Hilbert problem for the so-called Moisil-Teodoresco system in the space $\mathbb{R}^{3}$, see [MT31] and elsewhere.

Let $\mathcal{X}$ be a bounded domain with smooth boundary in $\mathbb{R}^{3}$. Consider the stationary Maxwell equations

$$
\begin{aligned}
\operatorname{div} v & =0, \\
\operatorname{curl} v & =0
\end{aligned}
$$

for a vector field $v=\left(v^{1}, v^{2}, v^{3}\right)$ in $\mathcal{X}$, where

$$
\operatorname{curl} v=\left(-\partial_{2} v^{2}+\partial_{1} v^{3}, \partial_{2} v^{1}-\partial_{0} v^{3},-\partial_{1} v^{1}+\partial_{0} v^{2}\right) .
$$

Since the substitution $v=\nabla p$ reduces the Maxwell equations to the Laplace equation for the potential $p$ in $\mathcal{X}$, we subject $v$ to a boundary condition

$$
b_{0} v^{1}+b_{1} v^{2}+b_{2} v^{3}=u_{0}
$$

on $\partial \mathcal{X}$, where $b=\left(b_{0}, b_{1}, b_{2}\right)$ is a continuous function on $\partial \mathcal{X}$ with values in $\mathbb{R}^{3}$ satisfying $|b|=1$. We frame the Maxwell equations in the Moisil-Teodoresco system

$$
\begin{aligned}
\operatorname{div} v & =0, \\
\operatorname{grad} u^{4}+\operatorname{curl} v & =0
\end{aligned}
$$

in $\mathcal{X}$ for an unknown function $u=\left(v, u^{4}\right)$ with values in $\mathbb{R}^{4}$. We require $u^{4}$ to satisfy

$$
u^{4}=0
$$

on $\partial \mathcal{X}$. Since (5.1) implies $\Delta u^{4}=0$, we see by the maximum principle that $u^{4} \equiv 0$ in $\mathcal{X}$. Thus, we come back to the initial boundary value problem for $v$.

If one substitutes $-u_{4}$ for $u_{4}$ into (5.1) and multiplies the scalar differential equation containing $-\partial_{0} u_{4}$ by -1 then one obtains the standard form of the MoisilTeodoresco system. For this reason we consider the generalised Riemann-Hilbert problem

$$
\begin{aligned}
A_{0} \frac{\partial u}{\partial x^{0}}+A_{1} \frac{\partial u}{\partial x^{1}}+A_{2} \frac{\partial u}{\partial x^{2}} & =f \text { in } \mathcal{X} \\
\left(\begin{array}{cccc}
b_{0} & b_{1} & b_{2} & 0 \\
0 & 0 & 0 & 1
\end{array}\right) u & =u_{0} \text { on } \partial \mathcal{X}
\end{aligned}
$$

with $A_{0}=E_{4}$ and

$$
A_{1}=\left(\begin{array}{rrrr}
0 & 1 & 0 & 0 \\
-1 & 0 & 0 & 0 \\
0 & 0 & 0 & 1 \\
0 & 0 & -1 & 0
\end{array}\right), \quad A_{2}=\left(\begin{array}{rrrr}
0 & 0 & 1 & 0 \\
0 & 0 & 0 & -1 \\
-1 & 0 & 0 & 0 \\
0 & 1 & 0 & 0
\end{array}\right) .
$$

The above matrices satisfy the relations

$$
\begin{aligned}
A_{i} A_{j}+A_{j} A_{i} & =-2 \delta_{i j} E_{4}, \\
A_{j}^{*} & =-A_{j}
\end{aligned}
$$

for $i, j=1,2$. 
Lemma 5.1. For $b=\nu$, there is no $(2 \times 4)$-matrix $C(x)$ of continuous functions on the boundary, such that

$$
\operatorname{rank}\left(\begin{array}{c}
B(x) \\
C(x)
\end{array}\right)=4
$$

for all $x \in \partial \mathcal{X}$.

Proof. Assume that the vectors $\left(\nu_{0}, \nu_{1}, \nu_{2}, 0\right)$ and $(0,0,0,1)$ can be completed pointwise to a basis of $\mathbb{R}^{4}$ which depends continuously on $x \in \partial \mathcal{X}$. We are then readily in a position to use the Gram-Schmidt orthogonalisation and get an orthonormal basis

$$
\left\{\left(\nu_{0}, \nu_{1}, \nu_{2}, 0\right),(0,0,0,1),\left(c_{0}, c_{1}, c_{2}, 0\right),\left(d_{0}, d_{1}, d_{2}, 0\right)\right\},
$$

whose elements are still continuous functions on the boundary. The inward unit normal vector $\nu$ is orthogonal to the vector field $c=\left(c_{0}, c_{1}, c_{2}\right)$. This means that the function $c(x)$ with values in $\mathbb{R}^{3}$ actually determines a tangential vector field on the unit sphere $\mathbb{S}^{2}$ in $\mathbb{R}^{3}$. However, by the hedgehog theorem, there is a continuous tangential vector field (vanishing at no point) on the sphere $\mathbb{S}^{n}$ only in the case when $n$ is odd. In other words, there may not exist any continuous tangential vector field on $\partial \mathcal{X}$, for $n=2$. And this is a contradiction to the assumption that $\left(\nu_{0}, \nu_{1}, \nu_{2}, 0\right)$ and $(0,0,0,1)$ can be completed to a basis of $\mathbb{R}^{4}$ continuously depending on $x \in \partial \mathcal{X}$.

A familiar topological argument shows that Lemma 5.1 remains still true for those vectors $b(x)$ which are tangential to $\partial \mathcal{X}$ at no point $x \in \partial \mathcal{X}$.

If $C$ is allowed to be a $(3 \times 4)$-matrix, then it already possible to find a $C(x)$ which depends continuously on $x \in \partial \mathcal{X}$ and supplements $B(x)$ to a matrix of rank 4. Namely, set

$$
C^{0}=\left(\begin{array}{rr}
b_{0} & 0 \\
b_{1} & 0 \\
b_{2} & 0 \\
0 & 1
\end{array}\right), \quad C^{1}=\left(\begin{array}{rrrr}
b_{1} & -b_{0} & 0 & 0 \\
b_{2} & 0 & -b_{0} & 0 \\
0 & b_{2} & -b_{1} & 0
\end{array}\right), \quad C^{2}=\left(\begin{array}{lll}
b_{2} & -b_{1} & b_{0}
\end{array}\right)
$$

for $x \in \partial \mathcal{X}$.

Lemma 5.2. Let $b=\left(b_{0}, b_{1}, b_{2}\right)$ be a unit vector of continuous functions on the boundary. Then the sequence

$$
0 \longrightarrow \mathbb{C}^{2} \stackrel{C^{0}(x)}{\longrightarrow} \mathbb{C}^{4} \stackrel{C^{1}(x)}{\longrightarrow} \mathbb{C}^{3} \stackrel{C^{2}(x)}{\longrightarrow} \mathbb{C} \longrightarrow 0
$$

is exact for each $x \in \partial \mathcal{X}$.

Proof. A trivial verification shows that $C^{i} C^{i-1}=0$ for $i=1$, 2, i.e., the sequence is a complex. It remains to evaluate the Laplace operators of this complex. These are

$$
\begin{aligned}
\left(C^{0}\right)^{*} C^{0} & =E_{2}, \\
C^{0}\left(C^{0}\right)^{*}+\left(C^{1}\right)^{*} C^{1} & =E_{4}, \\
C^{1}\left(C^{1}\right)^{*}+\left(C^{2}\right)^{*} C^{2} & =E_{3}, \\
C^{2}\left(C^{2}\right)^{*} & =1,
\end{aligned}
$$

and the lemma follows.

On choosing $C=C^{1}$ and

$$
\begin{aligned}
C^{\text {adj }} & =B\left(\sigma^{1}(A)(\imath \nu)\right)^{*} \\
B^{\text {adj }} & =-C\left(\sigma^{1}(A)(\imath \nu)\right)^{*}
\end{aligned}
$$


we thus obtain the Green formula of Theorem 4.3 related to the Riemann-Hilbert problem 5.2).

\section{The Fredholm PRoperty}

By the above, problem (5.2) is closely related to the oblique derivative problem for the inhomogeneous Laplace equation. From this view point, the following result is not especially surprising.

Theorem 6.1. Problem (5.2) is Fredholm if and only if the vector $b=\left(b_{0}, b_{1}, b_{2}\right)$ is at no point tangential to the boundary.

For the proof we recall the construction of the vector product of two vectors $a, b \in \mathbb{R}^{3}$. If $a=\left(a_{0}, a_{1}, a_{2}\right)$ and $b=\left(b_{0}, b_{1}, b_{2}\right)$, then one defines

$$
a \times b=\left(a_{1} b_{2}-a_{2} b_{1}, a_{2} b_{0}-a_{0} b_{2}, a_{0} b_{1}-a_{1} b_{0}\right) .
$$

Lemma 6.2. Given any $a, b \in \mathbb{R}^{3}$, it follows that

1) $a \times b \perp a$ and $a \times b \perp b$;

2) $a \times b=-(b \times a)$.

We now turn to the proof of Theorem 6.1.

Proof. By Theorem 3.1 the boundary value problem 5.2$)$ is Fredholm if and only if

$$
\operatorname{det}\left(B\left(\sum_{i=0}^{2} \nu_{i}(x) A_{i}^{*}\right)\left(\sum_{j=0}^{2} \tau_{j}(x) A_{j}\right) B^{*}-\imath E_{2}\right) \neq 0
$$

for all $x \in \partial \mathcal{X}$ and tangential unit vectors $\tau(x)=\left(\tau_{0}(x), \tau_{1}(x), \tau_{2}(x)\right)$ for $\partial \mathcal{X}$ at $x$. Therefore, we have to show that (6.1) holds if and only if the pointwise scalar product $(b(x), \nu(x))$ does not vanish at any point of $\partial \mathcal{X}$. By abuse of notation, we write $b, \nu$ and $\tau$ for the values of these vectors at an arbitrary fixed boundary point $x$.

We evaluate the left hand side of (6.1). It is

$$
\begin{aligned}
& \operatorname{det}\left(B\left(\sum_{i=0}^{2} \nu_{i} A_{i}^{*}\right)\left(\sum_{j=0}^{2} \tau_{j} A_{j}\right) B^{*}-\imath E_{2}\right) \\
& =\operatorname{det}\left(B\left(\begin{array}{rrrr}
\nu_{0} & -\nu_{1} & -\nu_{2} & 0 \\
\nu_{1} & \nu_{0} & 0 & \nu_{2} \\
\nu_{2} & 0 & \nu_{0} & -\nu_{1} \\
0 & -\nu_{2} & \nu_{1} & \nu_{0}
\end{array}\right)\left(\begin{array}{rrrr}
\tau_{0} & \tau_{1} & \tau_{2} & 0 \\
-\tau_{1} & \tau_{0} & 0 & -\tau_{2} \\
-\tau_{2} & 0 & \tau_{0} & \tau_{1} \\
0 & \tau_{2} & -\tau_{1} & \tau_{0}
\end{array}\right) B^{*}-\imath E_{2}\right)
\end{aligned}
$$

and troublesome direct calculations using $(\nu, \tau)=0$ show that the left hand side reduces to

$$
\begin{gathered}
\left.\operatorname{det}\left(\begin{array}{cc}
(b, \nu)(b, \tau)+(b \times \nu, b \times \tau) & (b \times \nu, \tau) \\
(b \times \tau, \nu) & (\nu, \tau)
\end{array}\right)-\imath E_{2}\right) \\
=\operatorname{det}\left(\begin{array}{cc}
(b, \nu)(b, \tau)+(b \times \nu, b \times \tau)-\imath & (b \times \nu, \tau) \\
(b \times \tau, \nu) & (\nu, \tau)-\imath
\end{array}\right) \\
=-(1+(b \times \tau, \nu)(b \times \nu, \tau))-\imath((b, \nu)(b, \tau)+(b \times \nu, b \times \tau)) .
\end{gathered}
$$

We are left with the task to show that

$$
(1+(b \times \tau, \nu)(b \times \nu, \tau))+\imath((b, \nu)(b, \tau)+(b \times \nu, b \times \tau)) \neq 0
$$


is fulfilled for all tangential vectors $\tau$ with $|\tau|=1$ if and only if

$$
(b, \nu) \neq 0
$$

on all of $\partial \mathcal{X}$. If one uses the indirect proof, it suffices to show that

$$
(1+(b \times \tau, \nu)(b \times \nu, \tau))+\imath((b, \nu)(b, \tau)+(b \times \nu, b \times \tau))=0
$$

is fulfilled for a tangential vector $\tau$ of length one if and only if $(b, \nu)=0$ at a boundary point of $\mathcal{X}$.

We prove " $\Leftarrow$." Assume that $(b, \nu)=0$ holds at some point $x_{0} \in \partial \mathcal{X}$. Hence it follows that $b$ is a tangential vector at the point $x_{0}$. We choose a tangential vector $\tau^{2}$ with the property that $\left\{b, \tau^{2}, \nu\right\}$ constitutes an orthonormal basis of $\mathbb{R}^{3}$. Then we get

$$
\begin{aligned}
(1 & \left.+\left(b \times \tau^{2}, \nu\right)\left(b \times \nu, \tau^{2}\right)\right)+\imath\left((b, \nu)\left(b, \tau^{2}\right)+\left(b \times \nu, b \times \tau^{2}\right)\right) \\
& =\left(1+(\nu, \nu)\left(-\tau^{2}, \tau^{2}\right)\right)+\imath\left(0\left(b, \tau^{2}\right)+\left(-\tau^{2}, \nu\right)\right) \\
& =(1-1)+\imath 0 \\
& =0
\end{aligned}
$$

as desired.

We now establish the implication " $\Rightarrow$." Suppose there is a tangential vector $\tau^{2}$ of length one, such that

$$
\left(1+\left(b \times \tau^{2}, \nu\right)\left(b \times \nu, \tau^{2}\right)\right)+\imath\left((b, \nu)\left(b, \tau^{2}\right)+\left(b \times \nu, b \times \tau^{2}\right)\right)=0 .
$$

One has to show that $(b, \nu)=0$ at this point. Choose a tangential vector $\tau^{1}$ of length one with the property that $\left\{\tau^{1}, \tau^{2}, \nu\right\}$ forms an orthonormal basis of $\mathbb{R}^{3}$. Write $b=c_{1} \tau^{1}+c_{2} \tau^{2}+c_{3} \nu$, where $c_{1}, c_{2}, c_{3} \in \mathbb{R}$. Then

$$
\begin{aligned}
1 & +\left(b \times \tau^{2}, \nu\right)\left(b \times \nu, \tau^{2}\right) \\
& =1+\left(\left(c_{1} \tau^{1}+c_{2} \tau^{2}+c_{3} \nu\right) \times \tau^{2}, \nu\right)\left(\left(c_{1} \tau^{1}+c_{2} \tau^{2}+c_{3} \nu\right) \times \nu, \tau^{2}\right) \\
& =1+\left(c_{1} \nu, \nu\right)\left(-c_{1} \tau^{2}, \tau^{2}\right) \\
& =1-c_{1}^{2}
\end{aligned}
$$

and

$$
\begin{aligned}
(b, & \nu)\left(b, \tau^{2}\right)+\left(b \times \nu, b \times \tau^{2}\right) \\
& =c_{3} c_{2}+\left(-c_{1} \tau^{2}+c_{2} \tau^{1}, c_{1} \nu-c_{3} \tau^{1}\right) \\
& =c_{3} c_{2}-c_{2} c_{3} \\
& =0 .
\end{aligned}
$$

By assumption, $1-c_{1}^{2}=0$ is valid. Hence it follows that $\left|c_{1}\right|=1$, and so $c_{2}=c_{3}=0$ and $(b, \nu)=\left(c_{1} \tau^{1}, \nu\right)=0$, as desired.

\section{Part 3. Boundary Fourier method}

\section{Hardy SPACES}

For a smooth function $f$ in $\mathcal{X}$, we set $f_{\varepsilon}(y):=f(y+\varepsilon \nu(y))$, where $\nu(y)$ is the inward unit normal vector to $\partial \mathcal{X}$ at $y$. So $f_{\varepsilon}$ is a family of smooth functions on $\partial \mathcal{X}$ parametrised by a small parameter $\varepsilon>0$. We say that $f$ admits a distribution boundary value on $\partial \mathcal{X}$, if

$$
\lim _{\varepsilon \rightarrow 0+} \int_{\partial \mathcal{X}} f_{\varepsilon} g d s=:\left\langle f_{0}, g\right\rangle
$$


exists for all $g \in C_{\text {comp }}^{\infty}(\partial \mathcal{X})$. In this case the limit defines a distribution $f_{0}$ on the boundary and the convergence is not only in the weak sense but also in the strong topology on $\mathcal{D}^{\prime}(\partial \mathcal{X})$.

The local structure of harmonic functions admitting distribution boundary values is well known.

Theorem 7.1. For a harmonic function $f$ in $\mathcal{X}$, the following properties are equivalent:

1) $f$ admits a distribution boundary value on $\partial \mathcal{X}$.

2) $f$ is in the Sobolev space $H^{-s}(\mathcal{X})$, for some integer $s$.

3) There exist an integer $N$ and $C>0$, such that $|f(x)| \leq C /(\operatorname{dist}(x, \partial \mathcal{X}))^{N}$ for all $x \in \mathcal{X}$.

4) For any $x_{0} \in \partial \mathcal{X}$ there are a neighbourhood $U$ in $\mathbb{R}^{n}$ and a function $h$ harmonic in $U \cap \mathcal{X}$ and continuous in $U \cap \overline{\mathcal{X}}$, such that $f=\langle c, \partial\rangle^{N} h$ in $U \cap \mathcal{X}$, where $c \in \mathbb{R}^{n}$ is a constant vector and $N$ an integer.

Proof. See [Str84, Theorem 1.1]. The proof actually shows that, for a harmonic function $f$ which admits a distribution boundary value on $\partial \mathcal{X}$, this boundary value is the trace of $f$ on $\partial \mathcal{X}$ in the sense of Sobolev spaces, i.e., $f_{0} \in H^{-s-1 / 2}(\partial \mathcal{X})$ provided $f \in H^{-s}(\mathcal{X})$.

From Theorem 7.1 it follows that if $f$ is a harmonic function in $\mathcal{X}$ which admits a distribution boundary value on $\partial \mathcal{X}$ then

$$
\sum_{|\alpha| \leq m} b_{\alpha}(x) \partial^{\alpha} f
$$

also admits a distribution boundary value on $\partial \mathcal{X}$ whenever the coefficients $b_{\alpha}$ are $C^{\infty}$ in the closure of $\mathcal{X}$. It is worth pointing out that this function need not be harmonic.

Just as in the case of more familiar harmonic Hardy spaces, the Poisson integral mediates between boundary values and the corresponding harmonic functions. Given a distribution $f_{0}$ on $\partial \mathcal{X}$, we write $P\left(f_{0}\right)$ for the Poisson integral of $f_{0}$. It is defined by $P\left(f_{0}\right)(x)=\left\langle P(x, \cdot), f_{0}\right\rangle$ for $x \in \mathcal{X}$, where $P(x, y)$ is the Poisson kernel for $\mathcal{X}$, i.e., the normal derivative of the Green function $G(x, y)$ at $y \in \partial \mathcal{X}$. For each integer $s$, the Poisson integral induces an isomorphism of $H^{-s-1 / 2}(\partial \mathcal{X})$ onto the subspace of $H^{-s}(\mathcal{X})$ consisting of harmonic functions in $\mathcal{X}$. Its inverse is the map assigning to each harmonic function $f \in H^{-s}(\mathcal{X})$ its boundary value, see Corollary 1.7 in Str84.

Yet another designation for functions in $\mathcal{X}$, which are polynomially bounded in $1 / \operatorname{dist}(x, \partial \mathcal{X})$, is functions of finite order of growth near the boundary, cf. Chapter 9 in Tar95] and elsewhere. By the above, a harmonic function of finite order of growth near the boundary in $\mathcal{X}$ is uniquely determined by its distribution boundary value on $\partial \mathcal{X}$. This allows one to identify harmonic functions of finite order of growth in $\mathcal{X}$ with their boundary values on $\partial \mathcal{X}$. In this way we obtain many interesting Banach spaces of harmonic functions in the domain $\mathcal{X}$. The most popular of them is perhaps the Hardy space $\mathcal{H}^{2}(\mathcal{X})$. This space is defined to consist of all harmonic functions in $\mathcal{X}$ of finite order of growth near the boundary, whose distribution boundary values on $\partial \mathcal{X}$ belong to $L^{2}(\partial \mathcal{X})$. When endowed with $L^{2}(\partial \mathcal{X})$-norm, $\mathcal{H}^{2}(\mathcal{X})$ is a Hilbert space. 
Let now $u$ be a smooth function in $\mathcal{X}$ with values in $\mathbb{C}^{k}$ satisfying a generalised Cauchy-Riemann system $A u=0$ in $\mathcal{X}$. If there exist an integer $N$ and $C>0$, such that $|u(x)| \leq C /(\operatorname{dist}(x, \partial \mathcal{X}))^{N}$ for all $x \in \mathcal{X}$, then the same is true for the components of $u$. By Theorem 7.1, each component admits a distribution boundary value on $\partial \mathcal{X}$. Hence, $u$ admits a boundary value on $\partial \mathcal{X}$ which is a continuous linear functional on $C_{\text {comp }}^{\infty}\left(\partial \mathcal{X}, \mathbb{C}^{k}\right)$. Moreover, both $B u$ and $C u$ admit boundary values on $\partial \mathcal{X}$ which are distributions with values in $\mathbb{C}^{k}$. This is precisely the sense in which we interpret them in the following formula analogous to the Cauchy integral formula.

Let $e(x)$ be the standard fundamental solution of convolution type for $\Delta$, i.e., $e(x)=(2 \pi)^{-1} \log |x|$, if $n=2$, and

$$
e(x)=\frac{1}{\sigma_{n}} \frac{1}{2-n} \frac{1}{|x|^{n-2}},
$$

if $n \geq 3$, where $\sigma_{n}$ is the surface area of the unit sphere in $\mathbb{R}^{n}$. The matrix $\Phi=-A^{*} e$ is a (two-sided) fundamental solution of convolution type of the operator $A$, i.e., the fundamental equations $\Phi A=A \Phi=I$ hold on compactly supported distributions in $\mathbb{R}^{n}$ with values in $\mathbb{C}^{k}$.

Lemma 7.2. For each solution $u$ to equations $A u=0$ in $\mathcal{X}$ of finite order of growth near $\partial \mathcal{X}$, it follows that

$-\int_{\partial \mathcal{X}}\left(\left(B u, C^{\operatorname{adj}} \Phi(x-\cdot)^{*}\right)_{y}-\left(C u, B^{\operatorname{adj}} \Phi(x-\cdot)^{*}\right)_{y}\right) d s= \begin{cases}u(x), & \text { if } x \in \mathcal{X}, \\ 0, & \text { if } x \in \mathbb{R}^{n} \backslash \overline{\mathcal{X}} .\end{cases}$

Note that $(\Phi(x-y))^{*}=(A e)(x-y)$ for all $x$ and $y$ away from the diagonal of $\mathbb{R}^{n}$, as is easy to check.

Proof. See Theorem 9.4.1 of [Tar95].

This reasoning, when looked at from a more general point of view, leads to new investigations of Fredholm boundary value problems in Hardy spaces, see [Tar95, 11.2.2].

\section{The Cauchy problem}

For $u \in \mathcal{H}^{2}(\mathcal{X})^{k}$, the Green formula 7.2 displays the Cauchy data of $u$ on the boundary of $\mathcal{X}$ with respect to the operator $A$. These are weak limit values of $B u$ and $C u$ on $\partial \mathcal{X}$. Hence we formulate the Cauchy problem as follows: Given any $u_{0} \in L^{2}\left(\partial \mathcal{X}, \mathbb{C}^{l_{0}}\right)$ and $u_{1} \in L^{2}\left(\partial \mathcal{X}, \mathbb{C}^{l_{2}}\right)$, find a function $u \in \mathcal{H}^{2}(\mathcal{X})^{k}$ satisfying $A u=0$ in $\mathcal{X}$ and

$$
\left\{\begin{array}{l}
B u=u_{0}, \\
C u=u_{1}
\end{array}\right.
$$

on $\partial \mathcal{X}$. In order that there may exist a solution $u \in \mathcal{H}^{2}(\mathcal{X})^{k}$ to problem 8.1, it is necessary that there be a function $u \in L^{2}\left(\partial \mathcal{X}, \mathbb{C}^{k}\right)$ satisfying $B u=u_{0}$ and $C u=u_{1}$.

Lemma 8.1. Suppose $u_{0} \in L^{2}\left(\partial \mathcal{X}, \mathbb{C}^{l_{0}}\right)$ and $u_{1} \in L^{2}\left(\partial \mathcal{X}, \mathbb{C}^{l_{2}}\right)$. For the existence of a function $u \in L^{2}\left(\partial \mathcal{X}, \mathbb{C}^{k}\right)$ satisfying $B u=u_{0}$ and $C u=u_{1}$ it is necessary and sufficient that

$$
\begin{aligned}
& B T^{-1}\left(B^{*} u_{0}+C^{*} u_{1}\right)=u_{0} \\
& C T^{-1}\left(B^{*} u_{0}+C^{*} u_{1}\right)=u_{1}
\end{aligned}
$$


Proof. Necessity. From the equalities $B u=u_{0}$ and $C u=u_{1}$ on the boundary it follows that $u=T^{-1}\left(B^{*} u_{0}+C^{*} u_{1}\right)$. On substituting this formula into $B u=u_{0}$ and $C u=u_{1}$ we obtain (8.2).

Sufficiency. Set $u=T^{-1}\left(B^{*} u_{0}+C^{*} u_{1}\right)$. Then $u \in L^{2}\left(\partial \mathcal{X}, \mathbb{C}^{k}\right)$ satisfies $B u=u_{0}$ and $C u=u_{1}$, which is due to 8.2 .

The Cauchy problem for solutions of systems with injective symbol and data on the whole boundary was intensively studied in the 1960s. To a certain extent this study was motivated by the paper Cal63. The study of the Cauchy problem in Hardy spaces is motivated by the problem of analytic continuation, cf. Chapter 11 in [Tar95].

Theorem 8.2. Let $u_{0} \in L^{2}\left(\partial \mathcal{X}, \mathbb{C}^{l_{0}}\right)$ and $u_{1} \in L^{2}\left(\partial \mathcal{X}, \mathbb{C}^{l_{2}}\right)$. In order that there may be a solution $u \in \mathcal{H}^{2}(\mathcal{X})^{k}$ to $A u=0$ in $\mathcal{X}$ subject to 8.1), it is necessary and sufficient that $\left(u_{0}, u_{1}\right)$ would satisfy (8.2) and

$$
\int_{\partial \mathcal{X}}\left(\left(u_{0}, C^{\text {adj }} g\right)_{x}-\left(u_{1}, B^{\text {adj }} g\right)_{x}\right) d s=0
$$

for all $g \in \mathcal{S}_{A^{*}}(\overline{\mathcal{X}})$.

Proof. Necessity. If $u \in \mathcal{H}^{2}(\mathcal{X})^{k}$ is a solution of the Cauchy problem with data $u_{0}$, $u_{1}$, then $u_{0}=B u$ and $u_{1}=C u$ satisfy (8.2), which is due to Lemma 8.1. Moreover, by the Green formula,

$$
\begin{aligned}
& \int_{\partial \mathcal{X}}\left(\left(u_{0}, C^{\text {adj }} g\right)_{x}-\left(u_{1}, B^{\text {adj }} g\right)_{x}\right) d s=\int_{\partial \mathcal{X}}\left(\left(B u, C^{\text {adj } g}\right)_{x}-\left(C u, B^{\text {adj }} g\right)_{x}\right) d s \\
& =0
\end{aligned}
$$

for all $g \in \mathcal{S}_{A^{*}}(\overline{\mathcal{X}})$, as required.

Sufficiency. We introduce a function $U$ in $\mathcal{X} \backslash \partial \mathcal{X}$ with values in $\mathbb{C}^{k}$ by the Green-type integral

$$
U(x)=-\int_{\partial \mathcal{X}}\left(\left(u_{0}, C^{\operatorname{adj}} \Phi(x-\cdot)^{*}\right)_{y}-\left(u_{1}, B^{\operatorname{adj}} \Phi(x-\cdot)^{*}\right)_{y}\right) d s,
$$

where $x \in \mathcal{X} \backslash \partial \mathcal{X}$. An easy calculation using (5.3) shows that

$$
\left(u_{0}, C^{\operatorname{adj}} \Phi(x-\cdot)^{*}\right)_{y}-\left(u_{1}, B^{\operatorname{adj}} \Phi(x-\cdot)^{*}\right)_{y}=\Phi(x-\cdot)\left(\sigma^{1}(A)(\imath \nu) u_{b}\right)
$$

on $\partial \mathcal{X}$, where

$$
u_{b}=T^{-1}\left(B^{*} u_{0}+C^{*} u_{1}\right) .
$$

By 8.2, we get $B u_{b}=u_{0}$ and $C u_{b}=u_{1}$, and so $u_{b}$ is of class $L^{2}\left(\partial \mathcal{X}, \mathbb{C}^{k}\right)$ if and only if $u_{0}$ and $u_{1}$ belong to $L^{2}\left(\partial \mathcal{X}, \mathbb{C}^{l_{0}}\right)$ and $L^{2}\left(\partial \mathcal{X}, \mathbb{C}^{l_{2}}\right)$, respectively. Thus, formula 8.4 reduces to

$$
U=-\Phi *\left([\partial \mathcal{X}] \sigma^{1}(A)(\imath \nu) u_{b}\right)
$$

in $\mathcal{X} \backslash \partial \mathcal{X}$.

For each fixed $x \in \mathcal{X} \backslash \overline{\mathcal{X}}$, the columns of the matrix $\Phi(x-\cdot)^{*}$ belong to $\mathcal{S}_{A^{*}}(\overline{\mathcal{X}})$. Hence, 8.3 implies that $U$ vanishes in the complement of $\overline{\mathcal{X}}$.

Set $u=U\lceil\mathcal{X}$. We next prove that $u$ is the desired solution of the Cauchy problem. This is equivalent to saying that $u \in \mathcal{H}^{2}(\mathcal{X})^{k}$ and $A u=0$ in $\mathcal{X}, u \uparrow_{\partial \mathcal{X}}=u_{b}$ at $\partial \mathcal{X}$.

From the structure of the fundamental matrix $\Phi$ it follows immediately that $u$ belongs to $\mathcal{H}^{2}(\mathcal{X})^{k}$ and satisfies $A u=0$ in $\mathcal{X}$. Since $u_{b} \in L^{2}\left(\partial \mathcal{X}, \mathbb{C}^{k}\right)$, the jump of 
the double layer potential $\Phi\left([\partial \mathcal{X}] \sigma u_{b}\right)$ under crossing the surface $\partial \mathcal{X}$ from $\mathcal{X} \backslash \overline{\mathcal{X}}$ to $\mathcal{X}$ just amounts to $u_{b}$. This is true even for all distributions $u_{b}$ on $\partial \mathcal{X}$ taking their values in $\mathbb{C}^{k}$, see Theorem 10.1.5 in [Tar95]. For the square integrable densities $u_{b}$ the jump is understood in an appropriate sense including the $L^{2}\left(\partial \mathcal{X}, \mathbb{C}^{k}\right)$-norm. Summarising we conclude that $u \Upsilon_{\partial \mathcal{X}}=u_{b}$, for $U$ vanishes in $\mathcal{X} \backslash \overline{\mathcal{X}}$. This completes the proof.

\section{OPERATOR-THEORETIC FOUNDATIONS}

The operator-theoretic foundations of the method of Fischer-Riesz equations are elaborated in Tar95, 11.1]. It goes back at least as far as [PF50]. Here we adapt this method for studying the Hilbert boundary value problem for generalised Cauchy-Riemann equations.

Any solution of generalised Cauchy-Riemann equations in $\mathcal{X}$ is a $k$-column of harmonic functions in this domain. Therefore, the $k$-fold product of the Hardy space

$$
\mathcal{H}^{2}(\mathcal{X})^{k}=\underbrace{\mathcal{H}^{2}(\mathcal{X}) \times \ldots \times \mathcal{H}^{2}(\mathcal{X})}_{k \text { times }}
$$

fits well to constitute the domain of problem 4.1), where $f=0$. More precisely, denote by $H_{1}$ the vector space of all $u \in \mathcal{H}^{2}(\mathcal{X})^{k}$ satisfying $A u=0$ in $\mathcal{X}$. When endowed with the $L^{2}\left(\partial \mathcal{X}, \mathbb{C}^{k}\right)$-norm, this space is complete, i.e., a Hilbert space. The operator $B$ maps $H_{1}$ continuously into $H_{2}=L^{2}\left(\partial \mathcal{X}, \mathbb{C}^{l_{0}}\right)$, it need not be one-to-one or onto.

Let $H$ be the subspace of $L^{2}\left(\partial \mathcal{X}, \mathbb{C}^{l_{0}}\right) \times L^{2}\left(\partial \mathcal{X}, \mathbb{C}^{l_{2}}\right)$ consisting of all pairs $\left(u_{0}, u_{1}\right)$ satisfying 8.2. Obviously, this subspace is closed, and so it is a Hilbert space under the unitary structure induced from the Cartesian product. According to Lemma 8.1. the space $H$ just amounts to the image of $L^{2}\left(\partial \mathcal{X}, \mathbb{C}^{k}\right)$ by the mapping $u \mapsto(B u, C u)$.

Lemma 9.1. As defined above, the space $H$ coincides with the Cartesian product $H_{2} \times \operatorname{im} C$, where im $C$ stands for the range of $C: L^{2}\left(\partial \mathcal{X}, \mathbb{C}^{k}\right) \rightarrow L^{2}\left(\partial \mathcal{X}, \mathbb{C}^{l_{2}}\right)$.

Proof. From what has already been said it follows that $H$ is a subspace of $H_{2} \times \operatorname{im} C$. Hence, we shall have established the lemma if we prove that both $H_{2} \times\{0\}$ and $\{0\} \times \operatorname{im} C$ belong to $H$.

Pick an arbitrary $u_{0} \in L^{2}\left(\partial \mathcal{X}, \mathbb{C}^{l_{0}}\right)$. Since the Laplacian $T^{0}=B B^{*}$ at step 0 of the the compatibility complex of Lemma 4.2 is invertible at each point of $\partial \mathcal{X}$, we get $u_{0}=B u$, where $u=B^{*}\left(T^{0}\right)^{-1} u_{0}$ belongs to $L^{2}\left(\partial \mathcal{X}, \mathbb{C}^{k}\right)$. From the equality $C B^{*}=0$ we see that $C u=0$, and so the pair $\left(u_{0}, 0\right)=(B u, C u)$ belongs to $H$, as desired.

Consider now a pair $\left(0, u_{1}\right)$, where $u_{1}=C u$ for some $u \in L^{2}\left(\partial \mathcal{X}, \mathbb{C}^{k}\right)$. Without loss of generality we can assume that $B u=0$ on the boundary, for if not, we replace $u$ by $C^{*}\left(T^{2}\right)^{-1} u_{1}$, where $T^{2}=\left(C^{2}\right)^{*} C^{2}+C^{1}\left(C^{1}\right)^{*}$ is the Laplacian at step 2 of the compatibility complex of Lemma 4.2 . Then $\left(0, u_{1}\right)=(B u, C u)$ belongs to $H$, and so $\{0\} \times \operatorname{im} C$ lies in $H$.

Recall that the image of $L^{2}\left(\partial \mathcal{X}, \mathbb{C}^{k}\right)$ by $C=C^{1}$ coincides with the kernel of $C^{2}$ in $L^{2}\left(\partial \mathcal{X}, \mathbb{C}^{l_{2}}\right)$, which is a consequence of Lemma 4.2 . Therefore, the range of $C$ is a closed subspace of $L^{2}\left(\partial \mathcal{X}, \mathbb{C}^{l_{2}}\right)$, and so a Hilbert space with induced unitary structure. 
Consider the mapping $M: H_{1} \rightarrow H$ given by $M u=(B u, C u)$, which corresponds to the Cauchy problem for solutions of $A u=0$ in $\mathcal{X}$ with Cauchy data $B u=u_{0}$ and $C u=u_{1}$ on $\partial \mathcal{X}$. By the above, $M$ is continuous. From Theorem 8.2 it follows that $M$ has closed range.

Denote by $M^{*}: H \rightarrow H_{1}$ the operator that is adjoint to $M: H_{1} \rightarrow H$ in the sense of Hilbert spaces.

Lemma 9.2. The null-space im $M^{*}$ of the operator $M^{*}$ is separable in the topology induced from $H$.

Proof. This is true by the school fact that any subspace of a separable metric space is separable.

Let $\mathcal{S}_{A^{*}}(\overline{\mathcal{X}})$ stand for the space of all solutions to the formal adjoint system $A^{*} g=0$ on neighbourhoods of $\overline{\mathcal{X}}$. Since $A^{*}$ is elliptic, these are real analytic functions with values in $\mathbb{C}^{k}$.

Lemma 9.3. Assume that $g \in \mathcal{S}_{A^{*}}(\overline{\mathcal{X}})$. Then the couple $\left(C^{\text {adj }} g,-B^{\text {adj }} g\right)$ belongs to $\operatorname{im} M^{*}$.

Proof. Using formulas $(5.3)$ we see that the operator $B^{\text {adj }}$ factors through $C$, to wit, $B^{\text {adj }}=-C T^{-1}\left(\sigma^{1}(A)(\imath \nu)\right)^{*}$. Hence it follows that the couple $\left(C^{\text {adj }} g,-B^{\text {adj }} g\right)$ belongs to $H$.

It remains to prove that $\left(M u,\left(C^{\text {adj }} g,-B^{\text {adj }} g\right)\right)_{H}=0$ for all $u \in H_{1}$. By the Green formula, we get

$$
\begin{aligned}
\left(M u,\left(C^{\mathrm{adj}} g,-B^{\mathrm{adj}} g\right)\right)_{H} & =\int_{\partial \mathcal{X}}\left(\left(B u, C^{\mathrm{adj} g}\right)_{x}-\left(C u, B^{\mathrm{adj} g}\right)_{x}\right) d s \\
& =0,
\end{aligned}
$$

as desired.

The subspace of im $M^{*}$ consisting of all elements of the form $\left(C^{\text {adj }} g,-B^{\text {adj }} g\right)$, where $g \in \mathcal{S}_{A^{*}}(\overline{\mathcal{X}})$, is separable. Hence, there are many ways to choose a sequence $\left\{g_{i}\right\}_{i=1,2, \ldots}$ in $\mathcal{S}_{A^{*}}(\overline{\mathcal{X}})$, such that the system $\left\{\left(C^{\text {adj }} g_{i},-B^{\text {adj }} g_{i}\right)\right\}$ is complete in this subspace.

In Example 9.6 we will show some explicit sequences $\left\{g_{i}\right\}$ with this property. For the moment we fix one of such sequences.

Lemma 9.4. As defined above, the system $\left\{\left(C^{\mathrm{adj}} g_{i},-B^{\mathrm{adj}} g_{i}\right)\right\}_{i=1,2, \ldots}$ is complete in $\operatorname{im} M^{*}$.

Proof. Let $\mathcal{F}$ be a continuous linear functional on im $M^{*}$ vanishing on each element of the system $\left\{\left(C^{\text {adj }} g_{i},-B^{\text {adj }} g_{i}\right)\right\}$. Since im $M^{*}$ is a closed subspace of $H$, the Riesz representation theorem implies the existence of an element $\left(u_{0}, u_{1}\right) \in \operatorname{im} M^{*}$, such that the action of $\mathcal{F}$ on im $M^{*}$ consists in scalar multiplication with the element $\left(u_{0}, u_{1}\right)$. In particular,

$$
\begin{aligned}
\mathcal{F}\left(C^{\text {adj }} g_{i},-B^{\text {adj }} g_{i}\right) & =\int_{\partial \mathcal{X}}\left(\left(C^{\text {adj }} g_{i}, u_{0}\right)_{x}-\left(B^{\text {adj }} g_{i}, u_{1}\right)_{x}\right) d s \\
& =0
\end{aligned}
$$

for all $i=1,2, \ldots$. Since the system $\left\{\left(C^{\text {adj }} g_{i},-B^{\text {adj }} g_{i}\right)\right\}_{i=1,2 \ldots}$ is dense in the subspace of im $M^{*}$ consisting of all elements of the form $\left(C^{\text {adj }} g,-B^{\text {adj }} g\right)$, where 
$g \in \mathcal{S}_{A^{*}}(\overline{\mathcal{X}})$, we get

$$
\int_{\partial \mathcal{X}}\left(\left(u_{0}, C^{\mathrm{adj}} g\right)_{x}-\left(u_{1}, B^{\mathrm{adj}} g\right)_{x}\right) d s=0
$$

for all $g \in \mathcal{S}_{A^{*}}(\overline{\mathcal{X}})$. We now use Theorem 8.2 which says that there exists a function $u \in \mathcal{H}^{2}(\mathcal{X})^{k}$ such that $A u=0$ in $\mathcal{X}$ and $B u=u_{0}, C u=u_{1}$ at the boundary of $\mathcal{X}$. In other words, $\left(u_{0}, u_{1}\right)=M u$. Hence it follows that $\mathcal{F}(h)=(h, M u)_{H}=0$ for all $h \in \operatorname{im} M^{*}$. Thus, $\mathcal{F} \equiv 0$ and the standard application of the Hahn-Banach theorem completes the proof.

Write $P$ for the projection of $H=H_{2} \times \operatorname{im} C$ onto the first factor. The composition $P M=B$ acting from $H_{1}$ to $H_{2}$ just amounts to the operator of boundary value problem (4.1) with $f=0$ in the updated setting. More precisely, given any $u_{0} \in L^{2}\left(\partial \mathcal{X}, \mathbb{C}^{l_{0}}\right)$, find $u \in \mathcal{H}^{2}(\mathcal{X})^{k}$ satisfying $A u=0$ in $\mathcal{X}$ and $B u=u_{0}$ weakly on the boundary of $\mathcal{X}$. The following lemma expresses the most important property of the system $\left\{g_{i}\right\}$.

Lemma 9.5. The system $\left\{B^{\text {adj }} g_{i}\right\}_{i=1,2, \ldots}$ is complete in the image of $L^{2}\left(\partial \mathcal{X}, \mathbb{C}^{k}\right)$ by $C$ if and only if $P M$ is injective.

Proof. By the Hahn-Banach theorem, $\left\{B^{\text {adj }} g_{i}\right\}$ is complete in the image im $C$ of $L^{2}\left(\partial \mathcal{X}, \mathbb{C}^{k}\right)$ by $C$ if and only if any continuous linear functional $\mathcal{F}$ on $\operatorname{im} C$ vanishing on each element of the system, is zero. Pick such a functional $\mathcal{F}$. By the Riesz representation theorem there is a function $u_{1} \in \operatorname{im} C$ such that $\mathcal{F}(h)=\left(h, u_{1}\right)$ for all $h \in \operatorname{im} C$. Using Lemma 9.1 we see that $\left(0, u_{1}\right)$ belongs to the space $H$. So we get

$$
\begin{aligned}
\left(\left(0, u_{1}\right),\left(C^{\text {adj }} g_{i},-B^{\text {adj }} g_{i}\right)\right)_{H} & =-\overline{\left(B^{\text {adj }} g_{i}, u_{1}\right)_{L^{2}\left(\partial \mathcal{X}, \mathbb{C}^{l_{2}}\right)}} \\
& =-\overline{\mathcal{F}\left(B^{\text {adj }} g_{i}\right)} \\
& =\overline{\mathcal{F}\left(C T^{-1}\left(\sigma^{1}(A)(\imath \nu)\right)^{*} g_{i}\right)} \\
& =0
\end{aligned}
$$

for all $i=1,2, \ldots$. On applying Lemma 9.4 we deduce that the element $\left(0, u_{1}\right)$ belongs to the orthogonal complement of the subspace im $M^{*}$ in $H$. Since the operator $M$ has closed range, the orthogonal complement of im $M^{*}$ coincides with the range of $M$. Hence, there is a function $u \in \mathcal{H}^{2}(\mathcal{X})^{k}$ satisfying $A u=0$ in $\mathcal{X}$ and $B u=0, C u=u_{1}$ on $\partial \mathcal{X}$. If the operator $P M$ is injective, then $u=0$ whence $u_{1}=0$ and $\mathcal{F}=0$. Conversely, if the functional $\mathcal{F}$ is different from zero, then $u_{1}$ is not zero and so the operator $P M$ fails to be injective, which is precisely the desired conclusion.

After removing the elements which are linear combinations of the previous ones from the system $\left\{B^{\text {adj }} g_{i}\right\}_{i=1,2, \ldots}$, we get a sequence $\left\{g_{i_{n}}\right\}$ in $\mathcal{S}_{A^{*}}(\overline{\mathcal{X}})$, such that the system $\left\{B^{\text {adj }} g_{i_{n}}\right\}$ is linearly independent. Applying then the Gram-Schmidt orthogonalisation to the system $\left\{B^{\text {adj }} g_{i_{n}}\right\}$ in $L^{2}\left(\partial \mathcal{X}, \mathbb{C}^{l_{2}}\right)$, we obtain a new system $\left\{e_{n}\right\}_{n=1,2, \ldots}$ in $\mathcal{S}_{A^{*}}(\overline{\mathcal{X}})$, such that $\left\{B^{\text {adj }} e_{n}\right\}$ is an orthonormal system in $L^{2}\left(\partial \mathcal{X}, \mathbb{C}^{l_{2}}\right)$. Moreover, $\left\{B^{\text {adj }} e_{n}\right\}$ is an orthonormal basis in the image of $L^{2}\left(\partial \mathcal{X}, \mathbb{C}^{k}\right)$ by $C$, provided that $P M$ is injective. Note that the elements $e_{n}$ of the new system have explicit expressions through the elements $\left\{g_{i_{1}}, \ldots, g_{i_{n}}\right\}$ of the old system in the form of Gram's determinants. 
Example 9.6. Since $\mathcal{X}$ is a bounded domain with smooth boundary, its complement has only finitely many connected components. Let $\left\{x_{i}\right\}$ be a finite set of points in $\mathbb{R}^{n} \backslash \overline{\mathcal{X}}$, such that each connected component of $\mathbb{R}^{n} \backslash \overline{\mathcal{X}}$ contains at least one point $x_{i}$. Then the columns of the matrix $\partial^{\alpha} \Phi\left(x_{i}-\cdot\right)^{*}$ belong to $\mathcal{S}_{A^{*}}(\overline{\mathcal{X}})$ and the system $\left\{B^{\text {adj }} \partial^{\alpha} \Phi\left(x_{i}-\cdot\right)^{*}\right\}$ is complete in the subspace of $L^{2}\left(\mathcal{X}, \mathbb{C}^{l_{2}}\right)$ formed by elements of the type $B^{\text {adj }} g$ with $g \in \mathcal{S}_{A^{*}}(\overline{\mathcal{X}})$.

The proof of this fact actually repeats the reasoning of Example 11.4.14 in Tar95. Apparently the system of Example 9.6 is most convenient for numerical simulations.

\section{Part 4. Application to the Riemann-Hilbert problem}

\section{The Fischer-Riesz EQuations}

Let $\left\{g_{i}\right\}_{i=1,2, \ldots}$ be an arbitrary sequence in $\mathcal{S}_{A^{*}}(\overline{\mathcal{X}})$ with the property that the system $\left\{\left(C^{\text {adj }} g_{i},-B^{\text {adj }} g_{i}\right)\right\}$ is complete in $\operatorname{im~} M^{*}$. Applying the Gram-Schmidt orthogonalisation to the system $\left\{B^{\text {adj }} g_{i}\right\}$ in $L^{2}\left(\partial \mathcal{X}, \mathbb{C}^{l_{2}}\right)$, we obtain a new system $\left\{e_{n}\right\}_{n=1,2 \ldots}$ in $\mathcal{S}_{A^{*}}(\overline{\mathcal{X}})$, such that the system $\left\{B^{\text {adj }} e_{n}\right\}$ is orthonormal in the space $L^{2}\left(\partial \mathcal{X}, \mathbb{C}^{l_{2}}\right)$.

Given any $u_{1} \in L^{2}\left(\partial \mathcal{X}, \mathbb{C}^{l_{2}}\right)$, we denote by $k_{n}\left(u_{1}\right)$ the Fourier coefficients of $u_{1}$ with respect to the system $\left\{B^{\text {adj }} e_{n}\right\}$, i.e.,

$$
k_{n}\left(u_{1}\right)=\int_{\partial \mathcal{X}}\left(u_{1}, B^{\mathrm{adj}} e_{n}\right)_{y} d s
$$

for $n=1,2, \ldots$

Lemma 10.1. If $u \in \mathcal{H}^{2}(\partial \mathcal{X})^{k}$ satisfies $A u=0$ in $\mathcal{X}$, then

$$
k_{n}(C u)=\int_{\partial \mathcal{X}}\left(B u, C^{\mathrm{adj}} e_{n}\right)_{y} d s,
$$

where $n=1,2, \ldots$

Proof. Using Lemma 9.3 we obtain

$$
\begin{aligned}
k_{n}(C u) & =\int_{\partial \mathcal{X}}\left(C u, B^{\mathrm{adj}} e_{n}\right)_{y} d s+\left(M u,\left(C^{\mathrm{adj}} e_{n},-B^{\mathrm{adj}} e_{n}\right)\right)_{H} \\
& =\int_{\partial \mathcal{X}}\left(B u, C^{\mathrm{adj}} e_{n}\right)_{y} d s,
\end{aligned}
$$

as desired.

Thus, in order to find the Fourier coefficients of the data $C u$ on the boundary with respect to the system $\left\{B^{\operatorname{adj}_{n}} e_{n}\right\}$ in $L^{2}\left(\partial \mathcal{X}, \mathbb{C}^{l_{2}}\right)$, it suffices to know only the data $B u$ of problem 4.1 .

Theorem 10.2. Let $u_{0} \in L^{2}\left(\partial \mathcal{X}, \mathbb{C}^{l_{0}}\right)$. In order that there be $a u \in \mathcal{H}^{2}(\mathcal{X})^{k}$ such that $A u=0$ in $\mathcal{X}$ and $B u=u_{0}$ on $\partial \mathcal{X}$, it is necessary and sufficient that

1) $\sum_{n=1}^{\infty}\left|c_{n}\right|^{2}<\infty$, where $c_{n}=\int_{\partial \mathcal{X}}\left(u_{0}, C^{\mathrm{adj}} e_{n}\right)_{y} d s$, and ary.

2) $\int_{\partial \mathcal{X}}\left(u_{0}, C^{\mathrm{adj}} g\right)_{y} d s=0$ for all $g \in \mathcal{S}_{A^{*}}(\overline{\mathcal{X}})$ satisfying $B^{\mathrm{adj}} g=0$ on the bound- 
Proof. Necessity. Suppose there is a function $u \in \mathcal{H}^{2}(\mathcal{X})^{k}$ satisfying $A u=0$ in $\mathcal{X}$ and $B u=u_{0}$ at $\partial \mathcal{X}$. Then $c_{n}=k_{n}(C u)$ for all $n=1,2, \ldots$, which is due to Lemma 10.1. Applying the Bessel inequality yields

$$
\sum_{n=1}^{\infty}\left|c_{n}\right|^{2}=\sum_{n=1}^{\infty}\left|k_{n}(C u)\right|^{2} \leq \int_{\partial \mathcal{X}}|C u|^{2} d s<\infty
$$

and 1) is proved. On the other hand, 2) follows immediately from the Green formula.

Sufficiency. We now assume that 1) and 2) are satisfied. Condition 1) implies, by the Fischer-Riesz theorem, that the series

$$
u_{1}=\sum_{n=1}^{\infty} c_{n} B^{\text {adj }} e_{n}
$$

converges in the space $L^{2}\left(\partial \mathcal{X}, \mathbb{C}^{l_{2}}\right)$. Since the summands of series 10.1 belong to the image of $L^{2}\left(\partial \mathcal{X}, \mathbb{C}^{k}\right)$ by $C$ and the range of $C$ is closed, it follows that $u_{1} \in \operatorname{im} C$. Hence, the pair $\left(u_{0}, u_{1}\right)$ actually belongs to $H$. Obviously, $\left\{c_{n}\right\}_{n=1,2, \ldots}$ are the Fourier coefficients of $u_{1}$ with respect to the orthonormal system $\left\{B^{\text {adj }} e_{n}\right\}$ in $L^{2}\left(\partial \mathcal{X}, \mathbb{C}^{l_{2}}\right)$. In other words, we get $c_{n}=k_{n}\left(u_{1}\right)$ for all $n=1,2, \ldots$ On substituting formulas for $c_{n}$ from 1 ) into these equalities we arrive at the orthogonality relations

$$
\int_{\partial \mathcal{X}}\left(\left(u_{0}, C^{\mathrm{adj}} e_{n}\right)_{y}-\left(u_{1}, B^{\mathrm{adj}} e_{n}\right)_{y}\right) d s=0
$$

for $n=1,2, \ldots$, cf. 8.3 .

Our next goal is to prove that the pair $\left(u_{0}, u_{1}\right)$ is actually orthogonal to all elements of the system $\left\{\left(C^{\text {adj }} g_{i},-B^{\text {adj }} g_{i}\right)\right\}_{i=1,2, \ldots}$ in $H$, this latter being complete in im $M^{*}$. To do this, let us recall how the system $\left\{e_{n}\right\}$ has been obtained from the system $\left\{g_{i}\right\}$.

Even if the system $\left\{\left(C^{\text {adj }} g_{i},-B^{\text {adj }} g_{i}\right)\right\}$ is linearly independent in $H$, the system $\left\{B^{\text {adj }} g_{i}\right\}$ may have elements which are linear combinations of the previous ones in the space $L^{2}\left(\partial \mathcal{X}, \mathbb{C}^{l_{2}}\right)$. Such elements should be eliminated from the system before applying the Gram-Schmidt orthogonalisation. For example, suppose that, for some $i$, the equality

$$
B^{\text {adj }} g_{i}=\sum_{j=1}^{i-1} c_{i, j} B^{\text {adj }} g_{j}
$$

is fulfilled with suitable complex numbers $c_{i, j}$. Consider the function

$$
g_{i}^{\prime}=g_{i}-\sum_{j=1}^{i-1} c_{i, j} g_{j}
$$

which belongs to $\mathcal{S}_{A^{*}}(\overline{\mathcal{X}})$. Obviously, $\left(C^{\text {adj }} g_{i}^{\prime},-B^{\text {adj }} g_{i}^{\prime}\right)$ lies in im $M^{*}$ and satisfies $B^{\text {adj }} g_{i}^{\prime}=0$. It follows that

$$
g_{i}=\sum_{j=1}^{i-1} c_{i, j} g_{j}+g_{i}^{\prime} .
$$

All the other elements $\left(C^{\text {adj }} g_{i},-B^{\text {adj }} g_{i}\right)$, except for the eliminated ones, are expressed, by the contents of Gram-Schmidt orthogonalisation, as linear combinations of the elements $\left\{\left(C^{\text {adj }} e_{n},-B^{\text {adj }} e_{n}\right)\right\}_{n=1, \ldots, i}$. Thus, any element of the system 
$\left\{\left(C^{\text {adj }} g_{i},-B^{\text {adj }} g_{i}\right)\right\}$ has a unique expression through the elements of the system $\left\{\left(C^{\text {adj }} e_{n},-B^{\text {adj }} e_{n}\right)\right\}_{n=1,2, \ldots}$ in the form

$$
g_{i}=\sum_{n=1}^{i} c_{i, n} e_{n}+g_{i}^{\prime}
$$

where $g_{i}^{\prime} \in \mathcal{S}_{A^{*}}(\overline{\mathcal{X}})$ satisfies $B^{\text {adj }} g_{i}^{\prime}=0$ on the boundary $\partial \mathcal{X}$.

From equalities (10.2) and $(10.3)$ and condition 2) of the theorem it follows immediately that

$$
\begin{aligned}
& \left(\left(u_{0}, u_{1}\right),\left(C^{\text {adj }} g_{i},-B^{\text {adj }} g_{i}\right)\right)_{H} \\
& \quad=\sum_{n=1}^{i} c_{i, n}\left(\left(u_{0}, u_{1}\right),\left(C^{\text {adj }} e_{n},-B^{\text {adj }} e_{n}\right)\right)_{H}+\left(\left(u_{0}, u_{1}\right),\left(C^{\text {adj }} g_{i}^{\prime},-B^{\text {adj }} g_{i}^{\prime}\right)\right)_{H} \\
& \quad=0
\end{aligned}
$$

for all $i=1,2, \ldots$. Since the system $\left\{\left(C^{\text {adj }} g_{i},-B^{\text {adj }} g_{i}\right)\right\}_{i=1,2, \ldots}$ is complete in $\operatorname{im} M^{*}$, the element $\left(u_{0}, u_{1}\right)$ belongs to the orthogonal complement of this subspace in $H$. Using the lemma of operator kernel annihilator, we deduce that there exists a function $u \in H_{1}$ satisfying $M u=\left(u_{0}, u_{1}\right)$. In particular, $u \in \mathcal{H}^{2}(\mathcal{X})^{k}$ satisfies $A u=0$ in $\mathcal{X}$ and $B u=u_{0}$ on $\partial \mathcal{X}$, i.e., $u$ is the desired solution of boundary value problem (4.1).

The convergence of the series in 1) guarantees the stability of boundary value problem (4.1). Under this condition, the range of the mapping $P M$ is described in terms of continuous linear functionals on the space $H$, cf. 2), which is impossible in the general case.

Corollary 10.3. Under the hypotheses of Theorem 10.2, if moreover the homogeneous adjoint boundary value problem (4.5) has no smooth solutions in $\overline{\mathcal{X}}$ different from zero, then for problem (4.1) to possess a solution $u \in \mathcal{H}^{2}(\mathcal{X})^{k}$ it is necessary and sufficient that

$$
\sum_{n=1}^{\infty}\left|c_{n}\right|^{2}<\infty
$$

Proof. This follows immediately from Theorem 10.2 since condition 2) is automatically fulfilled.

\section{Regularisation OF SOlutions}

Note that the proof of Theorem 10.2 works without the assumption that the operator $P M$ in $H$ is injective. Our next objective will be to construct an approximate solution to the boundary value problem of 4.1) with $f=0$. To this end it is natural to assume that the corresponding homogeneous boundary value problem has only zero solution in the space $\mathcal{H}^{2}(\mathcal{X})^{k}$, i.e., the mapping $P M$ is injective. In this case the orthonormal system $\left\{B^{\text {adj }} e_{n}\right\}$ is actually complete in the image of $L^{2}\left(\partial \mathcal{X}, \mathbb{C}^{k}\right)$ by $C$. The orthonormal bases of this form are said to be special, cf. Tar95, 11.3].

For $x \in \mathcal{X} \backslash \partial \mathcal{X}$, we denote by $k_{n}\left(B^{\operatorname{adj}} \Phi(x-\cdot)^{*}\right)$ the $k$-row whose entries are the Fourier coefficients of the columns of the $\left(l_{2} \times k\right)$-matrix $B^{\text {adj }} \Phi(x-\cdot)^{*}$ with respect 
to the orthonormal basis $\left\{B^{\text {adj }} e_{n}\right\}_{n=1,2, \ldots}$ in the image of $L^{2}\left(\partial \mathcal{X}, \mathbb{C}^{k}\right)$ by $C$. More precisely, we set

$$
k_{n}\left(B^{\operatorname{adj}} \Phi(x-\cdot)^{*}\right)=\int_{\partial \mathcal{X}}\left(B^{\text {adj }} \Phi(x-\cdot)^{*}, B^{\text {adj }} e_{n}\right)_{y} d s
$$

for $n=1,2, \ldots$

Lemma 11.1. For $n=1,2, \ldots$, the coefficients $k_{n}\left(B^{\operatorname{adj}} \Phi(x-\cdot)^{*}\right)$ are analytic functions in $\mathcal{X} \backslash \partial \mathcal{X}$ with values in $\left(\mathbb{C}^{k}\right)^{*}$.

Proof. The assertion is obvious, for the fundamental solution $\Phi(x-y)$ is analytic away from the diagonal of $\mathcal{X} \times \mathcal{X}$.

Consider the following (Schwartz) kernels $R_{N}$ defined for $x \in \mathcal{X} \backslash \partial \mathcal{X}$ and $y$ in a neighbourhood of $\overline{\mathcal{X}}$ :

$$
R_{N}(x, y)=\Phi(x-y)-\sum_{n=1}^{N} k_{n}\left(B^{\mathrm{adj}} \Phi(x-\cdot)^{*}\right)^{*} e_{n}(y)^{*},
$$

where $N=1,2, \ldots$

Lemma 11.2. As defined above, the kernels $R_{N}$ are analytic in $x \in \mathcal{X} \backslash \partial \mathcal{X}$ and $y$ in a neighbourhood of $\overline{\mathcal{X}}$ except for the diagonal $\{x=y\}$, and $A^{*}(y, D) R_{N}(\cdot-y)^{*}=0$ on this set.

Proof. This follows immediately from Lemma 11.1 and the fact that $e_{n} \in \mathcal{S}_{A^{*}}(\overline{\mathcal{X}})$, as desired.

The sequence $\left\{R_{N}\right\}$ provides a very special approximation of the fundamental solution $\Phi$.

Lemma 11.3. The sequence $\left\{B^{\text {adj }} R_{N}(x, \cdot)^{*}\right\}_{N=1,2, \ldots}$ converges to zero in the norm of $L^{2}\left(\partial \mathcal{X}, \mathbb{C}^{l_{2} \times k}\right)$ uniformly in $x$ on compact subsets of $\mathcal{X} \backslash \partial \mathcal{X}$.

Proof. In fact, we get

$$
\begin{aligned}
B^{\text {adj }} R_{N}(x, \cdot)^{*} & =B^{\text {adj }} \Phi(x-\cdot)^{*}-\sum_{n=1}^{N} B^{\text {adj }} e_{n} k_{n}\left(B^{\text {adj }} \Phi(x-\cdot)^{*}\right) \\
& =\sum_{n=N+1}^{\infty} B^{\text {adj }} e_{n} k_{n}\left(B^{\text {adj }} \Phi(x-\cdot)^{*}\right)
\end{aligned}
$$

for each fixed $x \in \mathcal{X} \backslash \partial \mathcal{X}$. The right-hand side of this equality is a remainder of the Fourier series of the element $B^{\text {adj }} R_{N}(x, \cdot)^{*}$ with respect to the orthonormal basis $\left\{B^{\text {adj }} e_{n}\right\}$ in the image of $L^{2}\left(\partial \mathcal{X}, \mathbb{C}^{k}\right)$ by $C$. Hence, it tends to zero in the $L^{2}\left(\partial \mathcal{X}, \mathbb{C}^{l_{2} \times k}\right)$-norm, as $N \rightarrow \infty$. This proves the first part of the lemma. The second part follows from a general remark on Fourier series, for the mapping of $\mathcal{X} \backslash \partial \mathcal{X}$ to $L^{2}\left(\partial \mathcal{X}, \mathbb{C}^{l_{2} \times k}\right)$ given by

$$
x \mapsto B^{\operatorname{adj}} \Phi(x-\cdot)^{*}
$$

is continuous.

The convergence of the approximations allows one to reconstruct solutions $u$ of the class $\mathcal{H}^{2}\left(\mathcal{X}, \mathbb{C}^{k}\right)$ to $A u=0$ in $\mathcal{X}$ through their data $B u$. 
Theorem 11.4. Every function $u \in \mathcal{H}^{2}(\mathcal{X})^{k}$ satisfying $A u=0$ in $\mathcal{X}$ can be represented by the integral formula

$$
u(x)=\lim _{N \rightarrow \infty}\left(-\int_{\partial \mathcal{X}}\left(B u, C^{\operatorname{adj}} R_{N}(x, \cdot)^{*}\right)_{y} d s\right)
$$

for all $x \in \mathcal{X}$.

Proof. Fix a point $x \in \mathcal{X}$. Since $R_{N}(x, \cdot)^{*}$ and $\Phi(x-\cdot)^{*}$ differ by a $k$-row of smooth solutions of the system $A^{*} g=0$ in a neighbourhood of $\overline{\mathcal{X}}$, one can write by the Green formula

$$
u(x)=-\int_{\partial \mathcal{X}}\left(\left(B u, C^{\text {adj }} R_{N}(x, \cdot)^{*}\right)_{y}-\left(C u, B^{\text {adj }} R_{N}(x, \cdot)^{*}\right)_{y}\right) d s
$$

for any $N=1,2, \ldots$. From $u \in \mathcal{H}^{2}(\mathcal{X})^{k}$ we deduce that $C u \in L^{2}\left(\partial \mathcal{X}, \mathbb{C}^{l_{2}}\right)$. Hence it follows by Lemma 11.3 that

$$
\lim _{N \rightarrow \infty} \int_{\partial \mathcal{X}}\left(C u, B^{\operatorname{adj}} R_{N}(x, \cdot)^{*}\right)_{y} d s=0 .
$$

Thus, letting $N \rightarrow \infty$ in 11.1 establishes the formula.

As mentioned, for many problems of mathematical physics formulas for approximate solution like that of Theorem 11.4 were earlier obtained by Kupradze and his colleagues, see Kup67.

\section{Solvability of Elliptic Boundary VALUE PROBLEMS}

We can now return to the Sobolev space setting of boundary value problem 4.1) which is $H_{1}=H^{1}\left(\mathcal{X}, \mathbb{C}^{k}\right)$. Given any $u \in H^{1}\left(\mathcal{X}, \mathbb{C}^{k}\right)$, both $A u$ and $B u$ are well defined in $L^{2}\left(\mathcal{X}, \mathbb{C}^{k}\right)$ and $H^{1 / 2}\left(\partial \mathcal{X}, \mathbb{C}^{l_{0}}\right)$, respectively. Hence, the analysis does not require any function spaces of negative smoothness. More generally, let $s$ be a natural number. Given any $u_{0}$ in $H^{s-1 / 2}\left(\partial \mathcal{X}, \mathbb{C}^{l_{0}}\right)$, we look for a $u \in H^{s}\left(\mathcal{X}, \mathbb{C}^{k}\right)$ satisfying (4.1). Theorem 10.2 still applies to establish the existence of a weak solution $u \in \mathcal{H}^{2}(\mathcal{X})^{k}$, provided that the conditions 1) and 2) are fulfilled. To infer the existence of a Sobolev space solution, one needs a regularity theorem for weak solutions in $\mathcal{H}^{2}(\mathcal{X})^{k}$ saying that any weak solution belongs actually to the Sobolev space $H^{s}\left(\mathcal{X}, \mathbb{C}^{k}\right)$. This is the case if 4.1 is an elliptic boundary value problem, i.e., the pair $\{A, B\}$ satisfies the Shapiro-Lopatinskij condition on the boundary of $\mathcal{X}$.

Corollary 12.1. Suppose a regularity theorem holds for boundary value problem (4.1). Let $u_{0} \in H^{s-1 / 2}\left(\partial \mathcal{X}, \mathbb{C}^{l_{0}}\right)$, where $s=1,2, \ldots$ Then, in order that there be $a u \in H^{s}\left(\mathcal{X}, \mathbb{C}^{k}\right)$ satisfying $A u=0$ in $\mathcal{X}$ and $B u=u_{0}$ on $\partial \mathcal{X}$ it is necessary and sufficient that

1) $\sum_{n=1}^{\infty}\left|c_{n}\right|^{2}<\infty$, where $c_{n}=\int_{\partial \mathcal{X}}\left(u_{0}, C^{\mathrm{adj}} e_{n}\right)_{y} d s$, and ary.

2) $\int_{\partial \mathcal{X}}\left(u_{0}, C^{\text {adj }} g\right)_{y} d s=0$ for all $g \in \mathcal{S}_{A^{*}}(\overline{\mathcal{X}})$ satisfying $B^{\text {adj }} g=0$ at the bound-

Proof. It is sufficient to prove the sufficiency of conditions 1) and 2). If the conditions 1) and 2) are satisfied, then there exists a function $u \in \mathcal{H}^{2}(\mathcal{X})^{k}$, such that $A u=0$ in $\mathcal{X}$ and $B u=u_{0}$ on $\partial \mathcal{X}$. For solutions of $A u=0$ in $\mathcal{X}$ the condition $u \in \mathcal{H}^{2}(\mathcal{X})^{k}$ just amounts to saying that $u \in H^{1 / 2}\left(\mathcal{X}, \mathbb{C}^{k}\right)$. Since 
$A u \in H^{s-1}\left(\mathcal{X}, \mathbb{C}^{k}\right)$ and $B u \in H^{s-1 / 2}\left(\partial \mathcal{X}, \mathbb{C}^{l_{0}}\right)$, the regularity theorem implies that $u \in H^{s}\left(\mathcal{X}, \mathbb{C}^{k}\right)$, as desired.

Assume that both the problem $\{A, B\}$ and its adjoint $\left\{A^{*}, B^{\text {adj }}\right\}$ with respect to the Green formula are elliptic. This is the case only if $l_{0}=l_{2}$, and so their common value amounts to $k / 2$. By the Fredholm property, the space of all $g \in \mathcal{S}_{A^{*}}(\overline{\mathcal{X}})$ satisfying $B^{\text {adj }} g=0$ on $\partial \mathcal{X}$, is finite dimensional. Moreover, the condition 2) alone is sufficient for the existence of a solution $u \in H^{s}\left(\mathcal{X}, \mathbb{C}^{k}\right)$ to problem 4.1). Hence it follows that for elliptic boundary value problems the condition 1) is automatically fulfilled.

Thus, the regularity problem for weak solutions of (4.1) is still of primary character in the study of boundary value problems. On the other hand, our approach demonstrates rather strikingly that Theorem 11.4 is of great importance for numerical simulation. 


\section{REFERENCES}

[Agr97] Agranovich, M. S., Elliptic Boundary Value Problems, In: Encyclopaedia of Mathematical Sciences, Vol. 79, Springer, Berlin et al., 1997, 1-144.

[AT13] Alsaedy, A., and Tarkhanov, N., The method of Fischer-Riesz equations for elliptic boundary value problems, J. of Complex Analysis 1 (2013), Issue 1, 1-11.

[AT16] Alsaedy, A., and Tarkhanov, N., A Hilbert boundary value problem for generalised Cauchy-Riemann equations, Advances in Applied Clifford Algebras 27 (2017), Issue 2, 931-953.

[Cal63] Calderón, A. P., Boundary value problems for elliptic equations, In: "Outlines Joint Symposium PDE" (Novosibisrk, 1963), Acad. Sci. USSR, Siberian Branch, Moscow, 1963, 303-304.

[Gak77] Gakhov, F. D., Boundary Value Problems, Nauka, Moscow, 1977.

[Kup67] Kupradze, V. D., Approximate solution of problems of mathematical physics, Uspekhi Mat. Nauk 22 (1967), No. 2, 59-107.

[MT31] Moisil, G. C., and Teodorescu, N., Fonction holomorphic dans l'espace, Bul. Soc. St. Cluj 6 (1931), 177-194.

[PF50] Picone, M., and Fichera, G., Neue funktional-analytische Grundlagen für die Existenzprobleme und Lösungsmethoden von Systemen linearer partieller Differentialgleichungen, Monatsh. Math. 54 (1950), 188-209.

[Ste91] Stern, I., Boundary value problems for generalized Cauchy-Riemann systems in the space, In: "Boundary value and initial value problems in complex analysis: Studies in complex analysis and its applications to partial differential equations, I" (Halle, 1988), Pitman Res. Notes Math. Ser., Vol. 256, Longman Sci. Tech., Harlow, 1991.

[Ste93a] Stern, I., On the existence of Fredholm boundary value problems for generalized Cauchy-Riemann systems, Complex Variables 21 (1993), 19-38.

[Ste93b] Stern, I., Direct methods for generalized Cauchy-Riemann systems in the space, Complex Variables 23 (1993), 73-100.

[Str84] Straube, E. J., Harmonic and analytic functions admitting a distribution boundary value, Ann. Scuola Norm. Super. Pisa 11 (1984), No. 4, 559-591.

[Tar95] Tarkhanov, N., The Cauchy Problem for Solutions of Elliptic Equations, Akademie Verlag, Berlin, 1995.

[Vol65] Volevich, L. R., On the solvability of boundary value problems for general elliptic systems, Mat. Sb. 68 (110) (1965), No. 3, 373-416.

Siberian Federal University, Institute of Mathematics and Computer Science, pr. Svobodnyi 79, 660041 Krasnoyarsk, Russia

E-mail address: paskaattt@yandex.ru

Institute of Mathematics, University of Potsdam, Karl-Liebknecht-Str. 24/25, 14476 Potsdam, Germany

E-mail address: tarkhanov@math.uni-potsdam.de 